\title{
Schmidt's subspace theorem for moving hypersurface targets
}

\author{
Nguyen Thanh Son, Tran Van Tan, and Nguyen Van Thin
}

\begin{abstract}
It was discovered that there is a formal analogy between Nevanlinna theory and Diophantine approximation. Via Vojta's dictionary, the Second Main Theorem in Nevanlinna theory corresponds to Schmidt's Subspace Theorem in Diophantine approximation. Recently, Cherry, Dethloff, and Tan (arXiv:1503.08801 v2 [math.CV]) obtained a Second Main Theorem for moving hypersurfaces intersecting projective varieites. In this paper, we shall give the counterpart of their Second Main Theorem in Diophantine approximation.
\end{abstract}

Keywords: Diophantine approximation, Schmidt's Subspace Theorem, Nevanlinna theory.

Mathematics Subject Classification 2010. 11J68, 11J25, 11J97.

\section{Introduction}

Let $k$ be an algebraic number field of degree $d$. Denote $M(k)$ by the set of places (i.e., equivalent classes of absolute values) of $k$ and write $M_{\infty}(k)$ for the set of Archimedean places. From $v \in M(k)$, we choose the normalized absolute value $|\cdot|_{v}$ such that $|\cdot|_{v}=|$.$| on \mathbb{Q}$ (the standard absolute value) if $v$ is archimedean, whereas for $v$ non-archimedean $|p|_{v}=p^{-1}$ if $v$ lies above the rational prime $p$. Denote by $k_{v}$ the completion of $k$ with respect to $v$ and by $d_{v}=\left[k_{v}: \mathbb{Q}_{v}\right]$ the local degree. We put $\|\cdot\|_{v}=|\cdot|_{v}^{d_{v} / d}$. Then norm $\|\cdot\|_{v}$ satisfies the following properties:

(i) $\|x\|_{v} \geq 0$, with equality if and only if $x=0$; 
(ii) $\|x y\|_{v}=\|x\|_{v}\|y\|_{v}$ for all $x, y \in k$;

(iii) $\left\|x_{1}+\cdots+x_{n}\right\|_{v} \leq B_{v}^{n_{v}} \cdot \max \left\{\left\|x_{1}\right\|_{v}, \ldots,\left\|x_{n}\right\|_{v}\right\}$ for all $x_{1}, \ldots, x_{n} \in k$, $n \in \mathbb{N}$, where $n_{v}=d_{v} / d, B_{v}=1$ if $v$ is non-archimedean and $B_{v}=n$ if $v$ is archimedean.

Moreover, for each $x \in k \backslash\{0\}$, we have the following product formula:

$$
\prod_{v \in M(k)}\|x\|_{v}=1 .
$$

For $v \in M(k)$, we also extend $\|\cdot\|_{v}$ to an absolute value on the algebraic closure $\bar{k}_{v}$.

For $x \in k$, the logarithmic height of $x$ is defined by $h(x)=\sum_{v \in M(k)} \log ^{+}\|x\|_{v}$, where $\log ^{+}\|x\|_{v}=\log \max \left\{\|x\|_{v}, 1\right\}$.

For $x=\left[x_{0}: \cdots: x_{M}\right] \in \mathbb{P}^{n}(k)$, we set $\|x\|_{v}=\max _{0 \leq i \leq M}\left\|x_{i}\right\|_{v}$, and define the logarithmic height of $x$ by

$$
h(x)=\sum_{v \in M(k)} \log \|x\|_{v}
$$

For a positive integer $d$, we set

$$
\mathcal{T}_{d}:=\left\{\left(i_{0}, \ldots, i_{M}\right) \in \mathbb{N}_{0}^{M+1}: i_{0}+\cdots+i_{M}=d\right\} .
$$

Let $Q$ be a homogeneous polynomial of degree $d$ in $k\left[x_{0}, \ldots, x_{M}\right]$. We write

$$
Q=\sum_{I \in \mathcal{T}_{d}} a_{I} x^{I} .
$$

Set $\|Q\|_{v}:=\max _{I}\left\|a_{I}\right\|_{v}$. The height of $Q$ is defined by

$$
h(Q):=\sum_{v \in M(k)} \log \|Q\|_{v} .
$$

For each $v \in M(k)$, the Weil function $\lambda_{Q, v}$ is defined by

$$
\lambda_{Q, v}(x):=\log \frac{\|x\|_{v}^{d} \cdot\|Q\|_{v}}{\|Q(x)\|_{v}}, \quad x \in \mathbb{P}^{M}(k) \text { such that } Q(x) \neq 0 .
$$

Let $\Lambda$ be an infinite index set. We call a moving hypersurface $Q$ in $\mathbb{P}^{M}(k)$ of degree $d$, indexed by $\Lambda$ each collection of polynomials $\{Q(\alpha)\}_{\alpha \in \Lambda}$ in 
$k\left[x_{0}, \ldots, x_{M}\right]$. Then, we can write $Q=\sum_{I \in \mathcal{T}_{d}} a_{I} x^{I}$, where $a_{I}$ 's are functions from $\Lambda$ into $k$ having no common zeros points.

Through this paper, we consider an infinite index $\Lambda$; a set $\mathcal{Q}:=\left\{Q_{1}, \ldots, Q_{q}\right\}$ of moving hypersurfaces in $\mathbb{P}^{M}(k)$, indexed by $\Lambda$; an arbitrary projective variety $V \subset \mathbb{P}^{M}(k)$ of dimension $n$ generated by the homogeneous ideal $\mathcal{I}(V)$. We write

$$
Q_{j}=\sum_{I \in \mathcal{T}_{d_{j}}} a_{j, I} x^{I}(j=1, \ldots, q) \text { where } d_{j}=\operatorname{deg} Q_{j} .
$$

Let $A \subset \Lambda$ be an infinite subset and denote by $(A, a)$ each set-theoretic map $a: A \rightarrow k$. Denote by $\mathcal{R}_{A}^{0}$ the set of equivalence classes of pairs $(C, a)$, where $C \subset A$ is a subset with finite complement and $a: C \rightarrow k$ is a map; and the equivalence relation is defined as follows: $\left(C_{1}, a_{1}\right) \sim\left(C_{2}, a_{2}\right)$ if there exists $C \subset C_{1} \cap C_{2}$ such that $C$ has finite complement in $A$ and $\left.a_{1}\right|_{C}=\left.a_{2}\right|_{C}$. Then $\mathcal{R}_{A}^{0}$ has an obvious ring structure. Moreover, we can embed $k$ into $\mathcal{R}_{A}^{0}$ as constant functions.

Definition 1.1. For each $j \in\{1, \ldots, q\}$, we write $\mathcal{T}_{d_{j}}=\left\{I_{j, 1}, \ldots, I_{j, M_{d_{j}}}\right\}$, where $M_{d_{j}}:=\left(\begin{array}{c}d_{j}+M \\ M\end{array}\right)$. A subset $A \subset \Lambda$ is said to be coherent with respect to $\mathcal{Q}$ if for every polynomial $P \in k\left[x_{1,1}, \ldots, x_{1, M_{d_{1}}}, \ldots, x_{q, 1}, \ldots, x_{q, M_{d_{q}}}\right]$ that is homogeneous in $x_{j, 1}, \ldots, x_{j, M_{d_{j}}}$ for each $j=1, \ldots, q$, either $P\left(a_{1, I_{1,1}}(\alpha), \ldots, a_{1, I_{1, M_{d_{1}}}}(\alpha), \ldots, a_{q, I_{q, 1}}(\alpha), \ldots, a_{q, I_{q, M_{d_{q}}}}(\alpha)\right)$ vanishes for all $\alpha \in$ $A$ or it vanishes for only finitely many $\alpha \in A$.

By [14, Lemma 2.1 there exists an infinite coherent subset $A \subset \Lambda$ with respect to $\mathcal{Q}$. For each $j \in\{1, \ldots, q\}$, we fix an index $I_{j} \in \mathcal{T}_{d_{j}}$ such that $a_{j, I_{j}} \not \equiv 0$ (this means that $a_{j, I_{j}}(\alpha) \neq 0$ for all, but finitely many, $\alpha \in A$ ), then $\frac{a_{j, I}}{a_{j, I_{j}}}$ defines an element of $\mathcal{R}_{A}^{0}$ for any $I \in \mathcal{T}_{d_{j}}$. This element given by the following function:

$$
\left\{\alpha \in A: a_{j, I_{j}}(\alpha) \neq 0\right\} \rightarrow k, \quad \alpha \mapsto \frac{a_{j, I}(\alpha)}{a_{j, I_{j}}(\alpha)} .
$$

Moreover, by coherent, the subring of $\mathcal{R}_{A}^{0}$ generated over $k$ by such all elements is an integral domain (p.3, [10]). We define $\mathcal{R}_{A, \mathcal{Q}}$ to be the field of fractions of that integral domain.

Denote by $\mathcal{A}$ the set of all functions $\left\{\alpha \in A: a_{j, I_{j}}(\alpha) \neq 0\right\} \rightarrow k, \quad \alpha \mapsto$ $\frac{a_{j, I}(\alpha)}{a_{j, I_{j}}(\alpha)}$ and $k_{\mathcal{Q}}$ the set of all formal finite sum $\sum_{m=1}^{s} t_{m} \prod_{i=1}^{s} c_{i}^{n_{i}}$, where $t_{m} \in k$, 
$c_{i} \in \mathcal{A}, n_{i} \in \mathbb{N}$. Each pair $(\widehat{b}, \widehat{c}) \in k_{\mathcal{Q}}^{2},(\hat{c}(\alpha) \neq 0$ for all, but finitely many, $\alpha \in A)$ defines a set-theoretic function, denoted by $\frac{\hat{b}}{\hat{c}}$, from $\{\alpha: \widehat{c}(\alpha) \neq 0\}$ to $k, \frac{\widehat{b}}{\hat{c}}(\alpha):=\frac{\widehat{b}(\alpha)}{\widehat{c}(\alpha)}$. Denote by $\widehat{\mathcal{R}}_{A, \mathcal{Q}}$ the set of all such functions. Each element $a \in \mathcal{R}_{A, \mathcal{Q}}$ is a class of some functions $\widehat{a}$ in $\widehat{\mathcal{R}}_{A, \mathcal{Q}}$. We call that $\widehat{a}$ is a special representative of $a$. It is clear that for any two special representatives $\widehat{a}_{1}, \widehat{a}_{2}$ of the same element $a \in \mathcal{R}_{A, \mathcal{Q}}$, we have $\widehat{a}_{1}(\alpha)=\widehat{a}_{2}(\alpha)$ for all, but finitely many $\alpha \in A$. For a polynomial $P:=\sum_{I} a_{I} x^{I} \in \mathcal{R}_{A, \mathcal{Q}}\left[x_{0}, \ldots, x_{M}\right]$, assume that $\widehat{a}_{I}$ is a special representative of $a_{I}$. Then $\widehat{P}:=\sum_{I} \hat{a}_{I} x^{I}$ is called a special representative of $P$. For each $\alpha \in A$ such that all functions $\widehat{a}_{I}$ 's are well defined at $\alpha$, we set $\widehat{P}(\alpha):=\sum_{\widehat{P}_{I}} \hat{a}_{I}(\alpha) x^{I} \in k\left[x_{0}, \ldots, x_{M}\right]$; and we also say that the special representative $\widehat{P}$ is well defined at $\alpha$. Note that each special representative $\widehat{P}$ of $P$ is well defined at all, but finitely many, $\alpha \in A$. If $\widehat{P}_{1}, \widehat{P}_{2}$ are two special presentatives of $P$, then $\widehat{P}_{1}(\alpha)=\widehat{P}_{2}(\alpha)$ for all, but finitely many $A$.

Definition 1.2. A sequence of points $x=\left[x_{0}: \cdots: x_{M}\right]: \Lambda \rightarrow V$ is said to be $V$-algebraically non-degenerate with respect to $\mathcal{Q}$ if for each infinite coherent subset $A \subset \Lambda$ with respect to $\mathcal{Q}$, there is no homogeneous polynomial $P \in \mathcal{R}_{A, \mathcal{Q}}\left[x_{0}, \ldots, x_{M}\right] \backslash \mathcal{I}_{A, \mathcal{Q}}(V)$ such that $\widehat{P}(\alpha)\left(x_{0}(\alpha), \ldots, x_{M}(\alpha)\right)=0$ for all, but finitely many, $\alpha \in A$ for some (then for all) representative $\widehat{P}$ of $P$, where $\mathcal{I}_{A, \mathcal{Q}}(V)$ is the ideal in $\mathcal{R}_{A, \mathcal{Q}}\left[x_{0}, \ldots, x_{M}\right]$ genarated by $\mathcal{I}(V)$.

Definition 1.3. We say that the family moving hypersurfaces $\mathcal{Q}$ is $V$-admissible if for each $1 \leq j_{0}<\cdots<j_{n} \leq q$, the system of equations

$$
Q_{j_{i}}(\alpha)\left(x_{0}, \ldots, x_{M}\right)=0, \quad 0 \leq i \leq n
$$

has no solution $\left(x_{0}, \ldots, x_{M}\right)$ satisfying $\left(x_{0}: \cdots: x_{M}\right) \in V(\bar{k})$, for all, but finitely many $\alpha \in \Lambda$, where $\bar{k}$ is the algebraic closure of $k$.

In 1997, Ru-Vojta [14] established the following Schmidt subspace theorem for the case of moving hyperplanes in projective spaces.

Theorem A. Let $k$ be a number field and let $S \subset M(k)$ be a finite set containing all archimedean places. Let $\Lambda$ be an infinite index set and let $\mathcal{H}:=\left\{H_{1}, \ldots, H_{q}\right\}$ be a set of moving hyperplanes in $\mathbb{P}^{M}(k)$, indexed by $\Lambda$. Let $x=\left[x_{0}: \cdots: x_{M}\right]: \Lambda \rightarrow \mathbb{P}^{M}(k)$ be a sequence of points. Assume that 
(i) $x$ is linearly nondegenerate with respect to $\mathcal{H}$, which mean, for each infinite coherent subset $A \subset \Lambda$ with respect to $\mathcal{H},\left.x_{0}\right|_{A}, \ldots,\left.x_{M}\right|_{A}$ are linearly independent over $\mathcal{R}_{A, \mathcal{H}}$,

(ii) $h\left(H_{j}(\alpha)\right)=o(h(x(\alpha)))$ for all $\alpha \in \Lambda$ and $j=1, \ldots, q$ (i.e. for all $\delta>0, h\left(H_{j}(\alpha)\right) \leqslant \delta h(x(\alpha))$ for all, but finitely many, $\left.\alpha \in \Lambda\right)$.

Then, for any $\varepsilon>0$, there exists an infinite index subset $A \subset \Lambda$ such that

$$
\sum_{v \in S} \max _{K} \sum_{J \in K} \lambda_{H_{j}(\alpha), v}(x(\alpha)) \leq(M+1+\varepsilon) h(x(\alpha))
$$

holds for all $\alpha \in A$. Here the maximum is taken over all subsets $K$ of $\{1, \ldots, q\}, \# K=M+1$ such that $H_{j}(\alpha), j \in K$ are linearly independent over $k$ for each $\alpha \in \Lambda$.

One of the most important developments in recent years in Diophantine approximation is Schmidt's subspace theorems for fixed hypersurfaces of Corvaja-Zannier [4] and Evertse-Ferretti [6, 7]. Motived by their paper, Min $\mathrm{Ru}$ [12, 13] obtained important results on the Second Main Theorem for fixed hypersurfaces. Later, Dethloff-Tan [5] and Cherry-Dethloff-Tan [3] generalized these Second Main Theorems of Min Ru to the case of moving results. Basing on method of Dethloff-Tan [5], Chen-Ru-Yan [2] and Le [9] extended Theorem A to the case of moving hypersurfaces in the projective spaces. In this paper, following the method of Cherry-Dethloff- Tan [3], we give the following counterpart of their Second Main Theorem in Diophantine approximation.

Theorem 1.4. Let $k$ be a number field and let $S \subset M(k)$ be a finite set containing all archimedean places. Let $x=\left[x_{0}: \cdots: x_{M}\right]: \Lambda \rightarrow V$ be a sequence of points. Assume that

(i) $\mathcal{Q}$ is $V$-admissible, and $x$ is $V$ - algebraically nondegenerate with respect to $\mathcal{Q}$;

(ii) $h\left(Q_{j}(\alpha)\right)=o(h(x(\alpha)))$ for all $\alpha \in \Lambda$ and $j=1, \ldots, q$ (i.e. for all $\delta>0, h\left(Q_{j}(\alpha)\right) \leqslant \delta h(x(\alpha))$ for all, but finitely many, $\left.\alpha \in \Lambda\right)$.

Then, for any $\varepsilon>0$, there exists an infinite index subset $A \subset \Lambda$ such that

$$
\sum_{v \in S} \sum_{j=1}^{q} \lambda_{Q_{j}(\alpha), v}\left(Q_{j}(x(\alpha)) \leq(n+1+\varepsilon) h(x(\alpha))\right.
$$

holds for all $\alpha \in A$. 
Remark 1.5. (i) By replacing $Q_{j}$ by $Q_{j}^{\frac{d}{d_{j}}}$, where $d=\operatorname{lcm}\left\{d_{1}, \ldots, d_{q}\right\}$, in Theorem 1.4, we may assume that $Q_{1}, \ldots, Q_{q}$ have the same degree $d$.

(ii) By replacing $Q_{j}=\sum_{I \in \mathcal{T}_{d}} a_{j, I} x^{I}$ by $Q_{j}^{\prime}=\sum_{I \in \mathcal{T}_{d}} \frac{a_{j I}}{a_{j I_{j}}} x^{I}$, in Theorem 1.4, we may assume that $Q_{j} \in \mathcal{R}_{A, \mathcal{Q}}\left[x_{0}, \ldots, x_{M}\right]$.

Acknowledgements: This research was supported by Vietnam National Foundation for Science and Technology Development (NAFOSTED) under grant number 101.02-2016.17. The second and the third named authors were partially supported by the Vietnam Institute for Advanced Studies in Mathematics. Tran Van Tan is currently Regular Associate Member of ICTP, Trieste, Italy. We would like to thank Gerd Dethloff for helpful comments on the first version of our paper.

\section{Some Lemmas}

We write

$$
Q_{j}=\sum_{I \in \mathcal{T}_{d}} a_{j I} x^{I}, \quad(j=0, \ldots, q) .
$$

Let $A \subset \Lambda$ be an infinity coherent subset with respect to $\mathcal{Q}$. For each $j \in$ $\{1, \ldots, q\}$, we fix an index $I_{j} \in \mathcal{T}_{d}$ such that $a_{j, I_{j}} \not \equiv 0$ (this means that $a_{j, I_{j}}(\alpha) \neq 0$ for all but finitely many $\left.\alpha \in A\right)$, then $\frac{a_{j I}}{a_{j I_{j}}}$ defines an element of $\mathcal{R}_{A}^{0}$ for any $I \in \mathcal{T}_{d}$. Set

$$
Q_{j}^{\prime}=\sum_{I \in \mathcal{T}_{d}} \frac{a_{j I}}{a_{j I_{j}}} x^{I}, \quad(j=0, \ldots, q) .
$$

Let $t=\left(\ldots, t_{j I}, \ldots\right)$ be a family of variables. Set

$$
\widetilde{Q_{j}}=\sum_{I \in \mathcal{T}_{d}} t_{j I} x^{I} \in k[t, x]
$$

We have $\widetilde{Q_{j}}\left(\ldots, \frac{a_{j I}}{a_{j I_{j}}}(\alpha), \ldots, x_{0}, \ldots, x_{M}\right)=Q_{j}^{\prime}(\alpha)\left(x_{0}, \ldots, x_{M}\right)$ for all $\alpha \in A$ outside a finite subset.

Assume that the ideal $\mathcal{I}(V)$ of $V$ is generated by homogeneous polynomials $P_{1}, \ldots, P_{m}$. Since $\mathcal{Q}$ is $V$-admissible, for each $J:=\left\{j_{0}, \ldots, j_{n}\right\} \subset$ 
$\{1, \ldots, q\}$ there is a subset $A_{J} \subset A$ with finite complement such that for all $\alpha \in A_{J}$ the homogeneous polynomials $P_{1}, \ldots, P_{m}, Q_{j_{0}}^{\prime}(\alpha), \ldots, Q_{j_{n}}^{\prime}(\alpha) \in$ $k\left[x_{0}, \ldots, x_{M}\right]$ have no common non-trivial solutions in $\bar{k}^{M+1}$. Denote by ${ }_{k[t]}\left(P_{1}, \ldots, P_{m}, \widetilde{Q}_{j_{0}}, \ldots, \widetilde{Q}_{j_{n}}\right)$ the ideal in the ring of polynomials in $x_{0}, \ldots, x_{M}$ with coefficients in $k[t]$ generated by $P_{1}, \ldots, P_{m}, \widetilde{Q}_{j_{0}}, \ldots, \widetilde{Q}_{j_{n}}$. A polynomial $\widetilde{R}$ in $k[t]$ is called an inertia form of the polynomials $P_{1}, \ldots, P_{m}, \widetilde{Q}_{j_{0}}, \ldots, \widetilde{Q}_{j_{n}}$ if it has the following property:

$$
x_{i}^{s} \cdot \widetilde{R} \in{ }_{k[t]}\left(P_{1}, \ldots, P_{m}, \widetilde{Q}_{j_{0}}, \ldots, \widetilde{Q}_{j_{n}}\right)
$$

for $i=0, \ldots, M$ and for some non-negative integer $s$ (see e.g. [16]).

It follows from the definition that the set $\mathcal{I}$ of inertia forms of polynomials $P_{1}, \ldots, P_{m}, \widetilde{Q}_{j_{0}}, \ldots, \widetilde{Q}_{j_{n}}$ is an ideal in $k[t]$.

It is well known that $(m+n+1)$ homogeneous polynomials $P_{i}\left(x_{0}, \ldots, x_{M}\right)$, $\widetilde{Q_{j}}\left(\ldots, t_{j I}, \ldots, x_{0}, \ldots, x_{M}\right), i \in\{1, \ldots, m\}, j \in J$ have no common nontrivial solutions in $x_{0}, \ldots, x_{M}$ for special values $t_{j I}^{0}$ of $t_{j I}$ if and only if there exists an inertia form $\widetilde{R}_{J}^{t_{j I}^{0}}$ such that $\widetilde{R}_{J}^{t_{j I}^{0}}\left(\ldots, t_{j I}^{0}, \ldots\right) \neq 0$ (see e.g. [16], page 254). For each $\alpha \in A_{J}$, choose $\widetilde{R}_{J}^{\alpha} \in \mathcal{I}$ with respect to the special values $t_{j I}^{\alpha}:=\frac{a_{j I}}{a_{j I_{j}}}(\alpha)$. Set $R_{J}^{\alpha}:=\widetilde{R}_{J}^{\alpha}\left(\ldots, \frac{a_{j I}}{a_{j I_{j}}}, \ldots\right)$. Then $R_{J}^{\alpha}$ is a special presentative of an element $\mathcal{R}_{A, \mathcal{Q}}$. By construction, we have

$$
R_{J}^{\alpha}(\alpha) \neq 0 \text { for all } \alpha \in A_{J} \text { (and hence, for all, but finitely many, } \alpha \in A \text { ). }
$$

Since $k[t]$ is Noetherian, $\mathcal{I}$ is generated by finite polynomials $\widetilde{R}_{J_{1}}, \ldots, \widetilde{R}_{J_{s}}$. For each $\alpha$, we write $\widetilde{R}_{J}^{\alpha}=\sum_{\ell=1}^{s} \widetilde{G}_{\ell}^{\alpha} \widetilde{R}_{J_{\ell}}, \widetilde{G}_{\ell}^{\alpha} \in k[t]$. We have that $G_{\ell}^{\alpha}:=$ $\widetilde{G}_{\ell}^{\alpha}\left(\ldots, \frac{a_{j I}}{a_{j I_{j}}}, \ldots\right)$, and $R_{J \ell}:=\widetilde{R}_{J \ell}\left(\ldots, \frac{a_{j I}}{a_{j I_{j}}}, \ldots\right)$ are special representatives of elements in $\mathcal{R}_{A, \mathcal{Q}}$. It is clear that $R_{J}^{\alpha}=\sum_{\ell=1}^{s} G_{\ell}^{\alpha} R_{J \ell}$. Hence, by (2.1), we have

$$
0 \neq R_{J}^{\alpha}(\alpha)=\sum_{\ell=1}^{s} G_{\ell}^{\alpha}(\alpha) R_{J_{\ell}}(\alpha)
$$

for all, but finitely many $\alpha \in A$. Therefore, there is $\ell_{0} \in\{1, \ldots, s\}$ such that

$$
R_{J_{\ell_{0}}}(\alpha) \neq 0 \quad \text { for all, but finitely many } \quad \alpha \in A .
$$


Furthermore, by the definition of the inertia forms, there are a non-negative integer $s$, polynomials $b_{i \ell} \in \mathcal{R}_{A, \mathcal{Q}}\left[x_{0}, \ldots, x_{M}\right]$ with $\operatorname{deg} b_{i j_{k}}=s-d$ and $\operatorname{deg} b_{i \ell}=s-\operatorname{deg} P_{\ell}$ such that

$$
R_{J_{\ell_{0}}} \cdot x_{i}^{s}=\sum_{k=0}^{n} b_{i j_{k}} \cdot Q_{j_{k}}^{\prime}+\sum_{\ell=1}^{m} b_{i \ell} \cdot P_{\ell},
$$

for all $0 \leq i \leq M$.

Let $x: \Lambda \rightarrow V \subset P^{M}(k)$ be a map. A map $(C, a) \in \mathcal{R}_{A}^{0}$ is called small with respect to $x$ if and only if

$$
h(a(\alpha))=o(h(x(\alpha)))
$$

which mean that, for every $\varepsilon>0$, there exists a subset $C_{\varepsilon} \subset C$ with finite complement such that $h(a(\alpha)) \leq \varepsilon h(x(\alpha))$ for all $\alpha \in C_{\varepsilon}$. We denote by $\mathcal{K}_{x}$ the set of all such small maps. Then, $\mathcal{K}_{x}$ is subring of $\mathcal{R}_{A}^{0}$. It is not an entire ring, however, if $(C, a) \in \mathcal{K}_{x}$ and $a(\alpha) \neq 0$ for all but finitely $\alpha \in C$, then we have $\left(C \backslash\{\alpha: a(\alpha)=0\}, \frac{1}{a}\right) \in \mathcal{K}_{x}$. Denote by $\mathcal{C}_{x}$ the set of all positive functions $g$ defined over $\Lambda$ outside a finite subset of $\Lambda$ such that

$$
\log ^{+}(g(\alpha))=o(h(x(\alpha))) \text {. }
$$

Then $\mathcal{C}_{x}$ is a ring. Moreover, if $(C, a) \in \mathcal{K}_{x}$, then for every $v \in M(k)$, the function $\|a\|_{v}: C \rightarrow \mathbb{R}^{+}$given by $\alpha \mapsto\|a(\alpha)\|_{v}$ belongs to $\mathcal{C}_{x}$. Furthermore, if $(C, a) \in \mathcal{K}_{x}$ and $a(\alpha) \neq 0$ for all but finitely $\alpha \in C$, the function $g$ : $\{\alpha \mid a(\alpha) \neq 0\} \mapsto \frac{1}{\|a(\alpha)\|_{v}}$ also belongs to $\mathcal{C}_{x}$.

From (2.2) and (2.3), similarly to Lemma 2.2 in [9], under the assumption of Theorem 1.4 and Remark 1.5, we have the following result.

Lemma 2.1. Let $A \subset \Lambda$ be coherent with respect to $\mathcal{Q}$. Then for each $J \subset$ $\{1, \ldots, q\}$, there are functions $l_{1, v}, l_{2, v} \in \mathcal{C}_{x}$ such that

$$
l_{2, v}(\alpha)\|x(\alpha)\|_{v}^{d} \leq \max _{j \in J}\left\|Q_{j}(\alpha)(x(\alpha))\right\|_{v} \leq l_{1, v}(\alpha)\|x(\alpha)\|_{v}^{d},
$$

for all $\alpha \in A$ ouside finite subset and all $v \in S$.

For each positive integer $\ell$ and for each vector sub-space $W$ in $k\left[x_{0}, \ldots, x_{M}\right]$ (or in $\mathcal{R}_{A, \mathcal{Q}}\left[x_{0}, \ldots, x_{M}\right]$ ), we denote by $W_{\ell}$ the vector space consisting of all holomogeneous polynomials in $W$ of degree $\ell$ (and of the zero polynomial). 
By the usual theory of Hilbert polynomials, for $N>>0$, we have

$$
\begin{aligned}
H_{V}(N) & :=\operatorname{dim}_{k} \frac{k\left[x_{0}, \ldots, x_{M}\right]_{N}}{\mathcal{I}(V)_{N}} \\
& =\operatorname{dim}_{\bar{k}} \frac{\bar{k}\left[x_{0}, \ldots, x_{M}\right]_{N}}{\mathcal{I}(V(\bar{k}))_{N}}=\operatorname{deg} V \cdot \frac{N^{n}}{n !}+O\left(N^{n-1}\right) .
\end{aligned}
$$

Definition 2.2. Let $W$ be a vector sub-space in $\mathcal{R}_{A, \mathcal{Q}}\left[x_{0}, \ldots, x_{M}\right]$. For each $\alpha \in A$, we denote

$W(\alpha):=\cup_{P \in W}\{\widehat{P}(\alpha): \widehat{P}$ is a special representative of $P$, well defined at $\alpha\}$.

It is clear that $W(\alpha)$ is a vector sub-space of $k\left[x_{0}, \ldots, x_{M}\right]$.

Lemma 2.3. Let $W$ be a vector sub-space in $\mathcal{R}_{A, \mathcal{Q}}\left[x_{0}, \ldots, x_{M}\right]_{N}$. Then two following assertions hold:

(i) There are $\gamma_{j} \in \mathcal{R}_{A, \mathcal{Q}}\left[x_{0}, \ldots, x_{M}\right]_{N}, j=1, \ldots, H$ such that $\gamma_{j}(\alpha), \ldots, \gamma_{H}(\alpha)$

form a basis of $W(\alpha)$, for all, but finitely many, $\alpha \in A$ (i.e. for any representative $\widehat{\gamma}_{j}$ of $\gamma_{j}$, then $\widehat{\gamma}_{j}(\alpha), \ldots, \widehat{\gamma}_{H}(\alpha)$ form a basis of $W(\alpha)$, for all, but finitely many, $\alpha \in A)$. In particular, dimension of $W(\alpha)$ does not depend on $\alpha \in A$ outside a finite subset.

(ii) Let $\left\{h_{j}\right\}_{j=1}^{K}$ be a basis of $W$. Then $\left\{h_{j}(\alpha)\right\}_{j=1}^{K}$ forms a basis of $W(\alpha)$ (hence, $\operatorname{dim}_{\mathcal{R}_{A, \mathcal{Q}}} W=\operatorname{dim}_{k} W(\alpha)$ ) for all, but finitely many, $\alpha \in A$ (i.e. for any special representative $\widehat{h}_{j}$ of $h_{j}$, then $\left\{\widehat{h}_{j}(\alpha)\right\}_{j=1}^{K}$ forms a basis of $W(\alpha)$ for all, but finitely many, $\alpha \in A$ ).

Proof. Set $H:=\max _{\alpha \in A} \operatorname{dim} W(\alpha)$. Taking $\alpha_{0} \in A$ such that $\operatorname{dim} W\left(\alpha_{0}\right)=$ $H$. Then, there are $\gamma_{j} \in \mathcal{R}_{A, \mathcal{Q}}\left[x_{0}, \ldots, x_{M}\right]_{N}(j=1, \ldots, H)$ and there are special representatives $\widehat{\gamma}_{j}$ of $\gamma_{j}(j=1, \ldots, H)$ such that $\left\{\widehat{\gamma}_{1}\left(\alpha_{0}\right), \ldots, \widehat{\gamma}_{H}\left(\alpha_{0}\right)\right\}$ form a basis of $W\left(\alpha_{0}\right)$.

Denote by $B$ the matrix of coefficients of $\left\{\widehat{\gamma}_{j}\right\}_{j=1}^{H}$. Then, $B\left(\alpha_{0}\right)$ has rank $H$. Hence, there is a square sub-matrix $B_{1}$ of $B$ with order $H$ such that $\operatorname{det} B_{1}\left(\alpha_{0}\right) \neq 0$. By coherent of $A$, there is a complement $A_{1}$ of a finite subset in $A$, such that det $B(\alpha) \neq 0$ and all coefficients of $\widehat{\gamma}_{j}$ 's are well defined at $\alpha$, for all $\alpha \in A_{1}$, Then, $\left\{\widehat{\gamma}_{1}(\alpha), \ldots, \widehat{\gamma}_{H}(\alpha)\right\}$ are linearly independent, for all $\alpha \in A_{1}$. On the other hand $\operatorname{dim} W(\alpha) \leqslant H$. Hence, $\left\{\widehat{\gamma}_{1}(\alpha), \ldots, \widehat{\gamma}_{H}(\alpha)\right\}$ is a basis of $W(\alpha)$ for all $\alpha \in A_{1}$. On the other hand, for any special representative $\widehat{\gamma}_{j}^{\prime}$ of $\gamma_{j}$, then $\widehat{\gamma}_{j}^{\prime}(\alpha)=\widehat{\gamma}_{j}(\alpha)$ for all, but finitely many, $\alpha \in A$. Hence, $\widehat{\gamma}_{1}^{\prime}(\alpha), \ldots, \widehat{\gamma}_{H}^{\prime}(\alpha)$ also form a basis of $W(\alpha)$ for all, but finitely many, $\alpha \in A$. This completes the proof of assertion (i). 
Let $\left(c_{i j}\right)$ be the matrix of coefficients of $\left\{h_{j}\right\}_{j=1}^{K}$. Since $\left\{h_{j}\right\}_{j=1}^{K}$ are linearly independent, there exists a square submatrix $C$ of $\left(c_{i j}\right)$ with order $K$ and $\operatorname{det} C \not \equiv 0$. Let $\widehat{c}_{i j}$ be an special representative of $c_{i j}$. Denote by $\widehat{C}$ the matrix which is defined from $C$ by replacing $c_{i j}$ by $\widehat{c}_{i j}$. Then $\operatorname{det} \widehat{C}$ is a special representative of $\operatorname{det} C$, and hence $\operatorname{det} \widehat{C} \not \equiv 0$. By coherent of $\mathrm{A}, \operatorname{det} \hat{C}(\alpha) \neq 0$ and all coefficients of $\widehat{h}_{j}^{\prime} s$ are well defined at $\alpha$, for all, but finitely many, $\alpha \in A$. By assertion (i), there are $\gamma_{j} \in \mathcal{R}_{A, \mathcal{Q}}\left[x_{0}, \ldots, x_{M}\right]_{N}, j=1, \ldots, H$ such that $\widehat{\gamma}_{1}(\alpha), \ldots, \widehat{\gamma}_{H}(\alpha)$ form a basis of $W(\alpha)$ for all, but finitely many, $\alpha \in A$, where $\widehat{\gamma}_{j}$ is a special representative of $\gamma_{j}$. We write $\gamma_{s}=\sum_{j=1}^{K} t_{s j} h_{j}$ with $t_{s j} \in \mathcal{R}_{A, \mathcal{Q}}$. Let $\widehat{t}_{s j}$ be a special representative of $t_{s j}(s \in\{1, \ldots, H\}, j \in$ $\{1, \ldots, K\})$. Then $\sum_{j=1}^{K} \widehat{t}_{s j} \widehat{h}_{j}$ is a special representative of $\gamma_{s}$. Hence, $\widehat{\gamma}_{s}(\alpha)=$ $\sum_{j=1}^{K} \widehat{t}_{s j}(\alpha) \widehat{h}_{j}(\alpha)(s=1, \ldots, H)$ for all, but finitely many, $\alpha \in A$. Combining with (i), we have that $\left\{\widehat{h}_{j}(\alpha)\right\}_{j=1}^{K}$ is a generating system of $W(\alpha)$ for all, but finitely many, $\alpha \in A$. On the other hand, since $\operatorname{det} \widehat{C}(\alpha) \neq 0$ and all coefficients of $\widehat{h}_{i j}^{\prime} s$ are well defined at $\alpha$, for all, but finitely many, $\alpha \in A$. Hence, $\widehat{h}_{1}(\alpha), \ldots, \widehat{h}_{K}(\alpha)$ are linearly independent for all, but finitely many, $\alpha \in A$. By these facts, $\left\{\widehat{h}_{1}(\alpha), \ldots, \widehat{h}_{K}(\alpha)\right\}$ is a basis of $W(\alpha)$, for all, but finitely many, $\alpha \in A$.

Denote by $\mathcal{I}_{A, \mathcal{Q}}(V)$ the ideal in $\mathcal{R}_{A, \mathcal{Q}}\left[x_{0}, \ldots, x_{M}\right]$ generated by the elements in $\mathcal{I}(V)$. It is clear that $\mathcal{I}_{A, \mathcal{Q}}(V)$ is also the sub-vector space of $\mathcal{R}_{A, \mathcal{Q}}\left[x_{0}, \ldots, x_{M}\right]$ generated by $\mathcal{I}(V)$.

We use the lexicographic order in $\mathbb{N}_{0}^{n}$ and for $I=\left(i_{1}, \ldots, i_{n}\right)$, set $\|I\|:=$ $i_{1}+\cdots+i_{n}$.

Definition 2.4. For each $I=\left(i_{1}, \cdots, i_{n}\right) \in \mathbb{N}_{0}^{n}$ and $N \in \mathbb{N}_{0}$ with $N \geq d\|I\|$, denote by $\mathcal{L}_{N}^{I}$ the set of all $\gamma \in \mathcal{R}_{A, \mathcal{Q}}\left[x_{0}, \ldots, x_{M}\right]_{N-d\|I\|}$ such that

$$
Q_{1}^{i_{1}} \cdots Q_{n}^{i_{n}} \gamma-\sum_{E=\left(e_{1}, \ldots, e_{n}\right)>I} Q_{1}^{e_{1}} \cdots Q_{n}^{e_{n}} \gamma_{E} \in \mathcal{I}_{A, \mathcal{Q}}(V)_{N} .
$$

for some $\gamma_{E} \in \mathcal{R}_{A, \mathcal{Q}}\left[x_{0}, \ldots, x_{M}\right]_{N-d\|E\|}$.

Denote by $\mathcal{L}^{I}$ the homogeneous ideal in $\mathcal{R}_{A, \mathcal{Q}}\left[x_{0}, \ldots, x_{M}\right]$ generated by $\cup_{N \geq d\|I\|} \mathcal{L}_{N}^{I}$.

Remark 2.5. i) $\mathcal{L}_{N}^{I}$ is a $\mathcal{R}_{A, \mathcal{Q}}$-vector sub-space of $\mathcal{R}_{A, \mathcal{Q}}\left[x_{0}, \ldots, x_{M}\right]_{N-d\|I\|}$, and $\left(\mathcal{I}(V), Q_{1}, \ldots, Q_{n}\right)_{N-d\|I\|} \subset \mathcal{L}_{N}^{I}$, where $\left(\mathcal{I}(V), Q_{1}, \ldots, Q_{n}\right)$ is the ideal in $\mathcal{R}_{A, \mathcal{Q}}\left[x_{0}, \ldots, x_{M}\right]$ generated by $\mathcal{I}(V) \cup\left\{Q_{1}, \ldots, Q_{n}\right\}$. 
ii) For any $\gamma \in \mathcal{L}_{N}^{I}$ and $P \in \mathcal{R}_{A, \mathcal{Q}}\left[x_{0}, \ldots, x_{M}\right]_{k}$, we have $\gamma \cdot P \in \mathcal{L}_{N+k}^{I}$

iii) $\mathcal{L}^{I} \cap \mathcal{R}_{A, \mathcal{Q}}\left[x_{0}, \ldots, x_{M}\right]_{N-d\|I\|}=\mathcal{L}_{N}^{I}$.

iv) $\frac{\mathcal{R}_{A, \mathcal{Q}}\left[x_{0}, \ldots, x_{M}\right]}{\mathcal{L}^{I}}$ is a graded modul over the graded ring $\mathcal{R}_{A, \mathcal{Q}}\left[x_{0}, \ldots, x_{M}\right]$.

Lemma 2.6. $\#\left\{\mathcal{L}^{I}: I \in \mathbb{N}_{0}^{n}\right\}<\infty$.

Proof. Suppose that $\#\left\{\mathcal{L}^{I}: I \in \mathbb{N}_{0}^{n}\right\}=\infty$. Then there exists an infinite sequence $\left\{\mathcal{L}^{I_{k}}\right\}_{k=1}^{\infty}$ consisting of pairwise different ideals. We write $I_{k}=$ $\left(i_{k 1}, \ldots, i_{k n}\right)$. Since $i_{k \ell} \in \mathbb{N}_{0}$, there exists an infinite sequence of positve integers $p_{1}<p_{2}<p_{3}<\cdots$ such that $i_{p_{1} \ell} \leqslant i_{p_{2} \ell} \leqslant i_{p_{3} \ell} \leqslant \cdots$, for all $\ell=1, \ldots, n$ : In fact, firstly we choose a sub-sequence $i_{q_{1} 1} \leqslant i_{q_{2} 1} \leqslant i_{q_{3} 1} \leqslant \cdots$ of $\left\{i_{k 1}\right\}_{k=1}^{\infty}$. Next, we choose a sub-sequence of $i_{r_{1} 2} \leqslant i_{r_{2} 2} \leqslant i_{r_{3} 2} \leqslant \cdots$ of $\left\{i_{q_{k}}\right\}_{k=1}^{\infty}$. Continuing the above process until obtaining a sub-sequence $i_{p_{1} n} \leqslant i_{p_{2} n} \leqslant i_{p_{3} n} \leqslant \cdots$.

We now prove that:

$$
\mathcal{L}^{I_{p_{1}}} \subset \mathcal{L}^{I_{p_{2}}} \subset \mathcal{L}^{I_{p_{3}}} \subset \cdots
$$

Indeed, for any $\gamma \in \mathcal{L}_{N}^{I_{p_{k}}}$ (for any $N$ and $k$ satisfying $N-\left\|I_{p_{k}}\right\| \geq 0$ ), we have

$$
Q_{1}^{i_{p_{k}} 1} \cdots Q_{n}^{i_{p_{k} n}} \gamma-\sum_{E=\left(e_{1}, \ldots, e_{n}\right)>I_{p_{k}}} Q_{1}^{e_{1}} \cdots Q_{n}^{e_{n}} \gamma_{E} \in \mathcal{I}_{\mathcal{R}_{A, \mathcal{Q}}}(V)_{N}
$$

for some $\gamma_{E} \in \mathcal{R}_{A, \mathcal{Q}}\left[x_{0}, \ldots, x_{M}\right]_{N-d\|E\|}$.

Then, since $i_{p_{k+1} 1}-i_{p_{k} 1}, \ldots, i_{p_{k+1} n}-i_{p_{k} n}$ are non-negative integers, we have $Q_{1}^{i_{p_{k+1} 1}} \cdots Q_{n}^{i_{p_{k+1} n}} \gamma-\sum_{E=\left(e_{1}, \ldots, e_{n}\right)>I_{p_{k}}} Q_{1}^{e_{1}+\left(i_{p_{k+1} 1^{1}}-i_{p_{k}}\right)} \cdots Q_{n}^{e_{n}+\left(i_{p_{k+1} n}-i_{p_{k} n}\right)} \gamma_{E} \in \mathcal{I}_{\mathcal{R}_{A, \mathcal{Q}}}(V)_{N}$.

On the other hand since $E=\left(e_{1}, \ldots, e_{n}\right)>I_{p_{k}}$ we have $\left(e_{1}+i_{p_{k+1} 1}-\right.$ $\left.i_{p_{k} 1}, \ldots, e_{n}+i_{p_{k+1} n}-i_{p_{k} n}\right)>I_{p_{k+1}}$. Therefore, $\gamma \in \mathcal{L}_{N-d\left\|I_{p_{k}}\right\|+d\left\|I_{p_{k+1}}\right\|}^{I_{p_{k+1}}}$. Hence, $\mathcal{L}_{N}^{I_{p_{k}}} \subset \mathcal{L}_{N-d\left\|I_{p_{k}}\right\|+d\left\|I_{p_{k+1}}\right\|}^{I_{p_{k+1}}}$ for all $k, N$. Therefore, $\mathcal{L}^{I_{p_{k}}} \subset \mathcal{L}^{I_{p_{k+1}}}$ for all $k$. We get (2.4).

Since $\mathcal{R}_{A, \mathcal{Q}}\left[x_{0}, \ldots, x_{M}\right]$ is a noetherian ring, the chain of ideals in (2.4) becomes finally stationary. This is a contradiction.

Set

$$
m_{N}^{I}:=\operatorname{dim}_{\mathcal{R}_{A, \mathcal{Q}}} \frac{\mathcal{R}_{A, \mathcal{Q}}\left[x_{0}, \ldots, x_{M}\right]_{N-d\|I\|}}{\mathcal{L}_{N}^{I}}
$$


For each positive integer $N$, denote by $\tau_{N}$ the set of all $I:=\left(i_{0}, \ldots, i_{n}\right) \in \mathbb{N}_{0}^{n}$ with $N-d\|I\| \geq 0$.

Let $\gamma_{I 1}, \ldots, \gamma_{I m_{N}^{I}} \in \mathcal{R}_{A, \mathcal{Q}}\left[x_{0}, \ldots, x_{M}\right]_{N-d\|I\|}$ such that they form a basis of the $\mathcal{R}_{A, \mathcal{Q}}-$ vector space $\frac{\mathcal{R}_{A, \mathcal{Q}}\left[x_{0}, \ldots, x_{M}\right]_{N-d\|I\|}}{\mathcal{L}_{N}^{I}}$.

Similarly to [3], Lemma 2.6, we have:

Lemma 2.7. $\left\{\left[Q_{1}^{i_{1}} \cdots Q_{n}^{i_{n}} \cdot \gamma_{I 1}\right], \ldots,\left[Q_{1}^{i_{1}} \cdots Q_{n}^{i_{n}} \cdot \gamma_{I m_{N}^{I}}\right], I=\left(i_{1}, \ldots, i_{n}\right) \in \tau_{N}\right\}$ is a basis of the $\mathcal{R}_{A, \mathcal{Q}}$-vector space $\frac{\mathcal{R}_{A, \mathcal{Q}}\left[x_{0}, \ldots, x_{M}\right]_{N}}{\mathcal{I}_{A, \mathcal{Q}}(V)_{N}}$.

Proof. Firstly, we prove that:

$$
\left\{\left[Q_{1}^{i_{1}} \cdots Q_{n}^{i_{n}} \cdot \gamma_{I 1}\right], \ldots,\left[Q_{1}^{i_{1}} \cdots Q_{n}^{i_{n}} \cdot \gamma_{I m_{N}^{I}}\right], I=\left(i_{1}, \ldots, i_{n}\right) \in \tau_{N}\right\}
$$

are linealy independent.

Indeed, for any $t_{I \ell} \in \mathcal{R}_{A, \mathcal{Q}},\left(I=\left(i_{1}, \ldots, i_{n}\right) \in \tau_{N}, \ell \in\left\{1, \ldots, m_{N}^{I}\right\}\right)$ such that

$$
\sum_{I \in \tau}\left(t_{I 1}\left[Q_{1}^{i_{1}} \cdots Q_{n}^{i_{n}} \cdot \gamma_{I 1}\right]+\cdots+t_{I m_{N}^{I}}\left[Q_{1}^{i_{1}} \cdots Q_{n}^{i_{n}} \cdot \gamma_{I m_{N}^{I}}\right]\right)=0
$$

Then

$$
\sum_{I \in \tau_{N}} Q_{1}^{i_{1}} \cdots Q_{n}^{i_{n}}\left(t_{I 1} \gamma_{I 1}+\cdots+t_{I m_{N}^{I}} \gamma_{I m_{N}^{I}}\right) \in \mathcal{I}_{A, \mathcal{Q}}(V)_{N}
$$

By the definition of $\mathcal{L}_{N}^{I}$, and by (2.6), we get

$$
t_{I^{*} 1} \gamma_{I^{*} 1}+\cdots+t_{I^{*} m_{N}^{I^{*}}} \gamma_{I^{*} m_{N}^{I^{*}}} \in \mathcal{L}_{N}^{I^{*}}
$$

where $I^{*}$ is the smallest elements of $\tau_{N}$.

On the other hand, $\left\{\gamma_{I^{*} 1}, \ldots, \gamma_{I^{*} m_{N}^{I^{*}}}\right\}$ makes a basis of $\frac{\mathcal{R}_{A, \mathcal{Q}}\left[x_{0}, \ldots, x_{M}\right]_{N-d\left\|I^{*}\right\|}}{\mathcal{L}_{N}^{I^{*}}}$.

Hence,

$$
t_{I^{*} 1}=\cdots=t_{I^{*} m_{N}^{I^{*}}}=0
$$

Then, by (2.6), we have

$$
\sum_{I \in \tau_{N} \backslash\left\{I^{*}\right\}} Q_{1}^{i_{1}} \cdots Q_{n}^{i_{n}}\left(t_{I 1} \gamma_{I 1}+\cdots+t_{I_{N}^{I}} \gamma_{I m_{N}^{I}}\right) \in \mathcal{I}_{A, \mathcal{Q}}(V)_{N} .
$$


Then, similarly to (2.7), we have

$$
t_{\tilde{I} 1}=\cdots=t_{\tilde{I} m_{N}^{\tilde{I}}}=0
$$

where $\tilde{I}$ is the smallest element of $\tau_{N} \backslash\left\{I^{*}\right\}$.

Continueting the above process, we get that $t_{I \ell}=0$ for all $I \in \tau_{N}$ and $\ell \in\left\{1, \ldots, m_{N}^{I}\right\}$, and hence, we get (2.5).

Denote by $\mathcal{L}$ the vector sub-space in $\mathcal{R}_{A, \mathcal{Q}}\left[x_{0}, \ldots, x_{M}\right]_{N}$ generated by

$$
\left\{Q_{1}^{i_{1}} \cdots Q_{n}^{i_{n}} \cdot \gamma_{I 1}, \ldots, Q_{1}^{i_{1}} \cdots Q_{n}^{i_{n}} \cdot \gamma_{I m_{N}^{I}}, I=\left(i_{1}, \ldots, i_{n}\right) \in \tau_{N}\right\}
$$

Now we prove that: For any $I=\left(i_{1}, \ldots, i_{n}\right) \in \tau_{N}$, we have

$$
Q_{1}^{i_{1}} \cdots Q_{n}^{i_{n}} \cdot \gamma_{I} \in \mathcal{L}+\mathcal{I}_{A, \mathcal{Q}}(V)_{N}
$$

for all $\gamma_{I} \in \mathcal{R}_{A, \mathcal{Q}}\left[x_{0}, \ldots, x_{M}\right]_{N-d\|I\|}$.

Set $I^{\prime}=\left(i_{1}^{\prime}, \ldots, i_{n}^{\prime}\right):=\max \left\{I: I \in \tau_{N}\right\}$. Since, $\gamma_{I^{\prime} 1}, \ldots, \gamma_{I^{\prime} m_{N}^{I^{\prime}}}$ form a basis of $\frac{\mathcal{R}_{A, \mathcal{Q}}\left[x_{0}, \ldots, x_{M}\right]_{N-d\|I\|}}{\mathcal{L}_{N}^{I^{\prime}}}$, for any $\gamma_{I^{\prime}} \in \mathcal{R}_{A, \mathcal{Q}}\left[x_{0}, \ldots, x_{M}\right]_{N-d\left\|I^{\prime}\right\|}$, we have

$$
\gamma_{I^{\prime}}=\sum_{\ell=1}^{m_{N}^{I^{\prime}}} t_{I^{\prime} \ell} \cdot \gamma_{I^{\prime} \ell}+h_{I^{\prime} \ell}, \text { where } h_{I^{\prime} \ell} \in \mathcal{L}_{N}^{I^{\prime}}, \text { and } t_{I^{\prime} \ell} \in \mathcal{R}_{A, \mathcal{Q}}
$$

On the other hand, by the defintion of $\mathcal{L}_{N}^{I^{\prime}}$, we have $Q_{1}^{i_{1}^{\prime}} \cdots Q_{n}^{i_{n}^{\prime}} \cdot h_{I^{\prime} \ell} \in$ $\mathcal{I}_{A, \mathcal{Q}}(V)_{N}$ (note that $\left.I^{\prime}=\max \left\{I: I \in \tau_{N}\right\}\right)$. Hence,

$$
Q_{1}^{i_{1}^{\prime}} \cdots Q_{n}^{i_{n}^{\prime}} \cdot \gamma_{I^{\prime}}=\sum_{\ell=1}^{m_{N}^{I^{\prime}}} t_{I^{\prime} \ell} Q_{1}^{i_{1}^{\prime}} \cdots Q_{n}^{i_{n}^{\prime}} \cdot \gamma_{I^{\prime} \ell}+Q_{1}^{i_{1}^{\prime}} \cdots Q_{n}^{i_{n}^{\prime}} \cdot h_{I^{\prime} \ell} \in \mathcal{L}+\mathcal{I}_{A, \mathcal{Q}}(V)_{N}
$$

We get (2.8) for the case where $I=I^{\prime}$.

Assume that (2.8) holds for all $I>I^{*}$. We prove that (2.8) holds also for $I=I^{*}=\left(i_{1}^{*}, \ldots, i_{n}^{*}\right)$.

Indeed, similarly to (2.9), for any $\gamma_{I^{*}} \in \mathcal{R}_{A, \mathcal{Q}}\left[x_{0}, \ldots, x_{M}\right]_{N-d\left\|I^{*}\right\|}$, we have

$$
\gamma_{I^{*}}=\sum_{\ell=1}^{m_{N}^{I^{*}}} t_{I^{*} \ell} \cdot \gamma_{I^{*} \ell}+h_{I^{*} \ell}, \text { where } h_{I^{*} \ell} \in \mathcal{L}_{N}^{I^{*}}, \text { and } t_{I^{*} \ell} \in \mathcal{R}_{A, \mathcal{Q}}
$$


Then,

$$
Q_{1}^{i_{1}^{*}} \cdots Q_{n}^{i_{n}^{*}} \cdot \gamma_{I^{*}}=\sum_{\ell=1}^{m_{N}^{I^{*}}} t_{I_{s} \ell} Q_{1}^{i_{1}^{*}} \cdots Q_{n}^{i_{n}^{*}} \cdot \gamma_{I^{*} \ell}+Q_{1}^{i_{1}^{*}} \cdots Q_{n}^{i_{n}^{*}} \cdot h_{I^{*} \ell}
$$

Since $h_{I^{*} \ell} \in \mathcal{L}_{N}^{I^{*}}$, we have

$$
Q_{1}^{i_{1}^{*}} \cdots Q_{n}^{i_{n}^{*}} \cdot h_{I^{*} \ell}=\sum_{E=\left(e_{1}, \ldots, e_{n}\right)>I^{*}} Q_{1}^{e_{1}} \cdots Q_{n}^{e_{n}} \cdot g_{E},
$$

for some $g_{E} \in \mathcal{R}_{A, \mathcal{Q}}\left[x_{0}, \ldots, x_{M}\right]_{N-d \cdot\|E\|}$.

Therefore, by the induction hypothesis,

$$
Q_{1}^{i_{1}^{*}} \cdots Q_{n}^{i_{n}^{*}} \cdot h_{I^{*} \ell} \in \mathcal{L}+\mathcal{I}_{A, \mathcal{Q}}(V)_{N}
$$

Then, by (2.10), we have

$$
Q_{1}^{i_{1}^{*}} \cdots Q_{n}^{i_{n}^{*}} \cdot \gamma_{I^{*}} \in \mathcal{L}+\mathcal{I}_{A, \mathcal{Q}}(V)_{N}
$$

This means that (2.8) holds for $I=I^{*}$. Hence, by induction we get (2.8).

For any $Q \in \mathcal{R}_{A, \mathcal{Q}}\left[x_{0}, \ldots, x_{M}\right]_{N}$, we write $Q=Q_{1}^{0} \cdots Q_{n}^{0} \cdot Q$. Then by (2.8), we have

$$
Q \in \mathcal{L}+\mathcal{I}_{A, \mathcal{Q}}(V)_{N}
$$

Hence,

$$
\left\{\left[Q_{1}^{i_{1}} \cdots Q_{n}^{i_{n}} \cdot \gamma_{I 1}\right], \ldots,\left[Q_{1}^{i_{1}} \cdots Q_{n}^{i_{n}} \cdot \gamma_{I m_{N}^{I}}\right], I=\left(i_{1}, \ldots, i_{n}\right) \in \tau_{N}\right\}
$$

is a generating system of $\frac{\mathcal{R}_{A, \mathcal{Q}}\left[x_{0}, \ldots, x_{M}\right]_{N}}{\mathcal{I}_{A, \mathcal{Q}}(V)_{N}}$. Combining with $(2.6)$, we get the conclution of Lemma 2.7.

Similarly to [3], Lemma, 2.8 we have:

Lemma 2.8. There are integers $n_{0}, c$ and $c^{\prime}$ such that the following assertions hold.

i) $\operatorname{dim}_{\mathcal{R}_{A, \mathcal{Q}}\left[x_{0}, \ldots, x_{M}\right]} \frac{\mathcal{R}_{A, \mathcal{Q}}\left[x_{0}, \ldots, x_{M}\right]\left[x_{0}, \ldots, x_{M}\right]_{N-d\|I\|}}{\left(\mathcal{I}(V), Q_{1}, \ldots, Q_{n}\right)_{N-d\|I\|}}=c$ for all $I \in \mathbb{N}_{0}^{n}, N \in \mathbb{N}_{0}$ satisfying $N-d\|I\| \geq n_{0}$.

ii) For each $I \in \mathbb{N}_{0}^{n}$ there is an integer $m^{I}$ such that $m^{I}=m_{N}^{I}$ for all $N \in \mathbb{N}_{0}$ satisfying $N-d\|I\| \geq n_{0}$.

iii) $m_{N}^{I} \leqslant c^{\prime}$, for all $I \in \mathbb{N}_{0}^{n}$ and $N \in \mathbb{N}_{0}$ satisfying $N-d \cdot\|I\| \geq 0$. 
Proof. Denote by $\left(\mathcal{I}(V), Q_{1}, \ldots, Q_{n}\right)$ the ideal in $\mathcal{R}_{A, \mathcal{Q}}\left[x_{0}, \ldots, x_{M}\right]$ generated by $\mathcal{I}(V) \cup\left\{Q_{1}, \ldots, Q_{n}\right\}$. For each $\alpha$ in $A$ such that all coefficients of $Q_{i}$ 's are well defined at $\alpha$, we denote by $\left(\mathcal{I}(V), Q_{1}(\alpha), \ldots, Q_{n}(\alpha)\right)$ the ideal in $k\left[x_{0}, \ldots, x_{M}\right]$ generated by $\mathcal{I}(V) \cup\left\{Q_{1}(\alpha), \ldots, Q_{n}(\alpha)\right\}$.

We have

$$
\left(\mathcal{I}(V), Q_{1}(\alpha), \ldots, Q_{n}(\alpha)\right) \subset\left(\mathcal{I}(V), Q_{1}, \ldots, Q_{n}\right)(\alpha) .
$$

Indeed, for any $P \in\left(\mathcal{I}(V), Q_{1}(\alpha), \ldots, Q_{n}(\alpha)\right)$, we write $P=G+Q_{1}(\alpha)$. $P_{1}+\cdots+Q_{n}(\alpha) \cdot P_{n}$, where $G \in \mathcal{I}(V)$, and $P_{i} \in k\left[x_{0}, \ldots, x_{M}\right]$. Take $P^{\prime} \in$ $\left(I(V), Q_{1}, \ldots, Q_{n}\right)$ which is defined by a presentation $\widehat{P}^{\prime}:=G+Q_{1} \cdot P_{1}+$ $\cdots+Q_{n} \cdot P_{n}$. It is clear that $\widehat{P}^{\prime}(\alpha)=P$. Hence, we get (2.11).

Let $I$ be an arbitrary element in $\tau_{N}$. Let $\left\{h_{k}:=\sum_{i=1}^{n} Q_{i} \cdot R_{i k}+\sum_{j=1}^{m_{k}} g_{j k}\right.$. $\left.\gamma_{j k}\right\}_{k=1}^{K}$ be a basis of $\left(\mathcal{I}(V), Q_{1}, \ldots, Q_{n}\right)_{N-d \cdot\|I\|}$, where $g_{j k} \in \mathcal{I}(V)$, and $R_{i k}, \gamma_{j k}, \in$ $\mathcal{R}_{A, \mathcal{Q}}\left[x_{0}, \ldots, x_{M}\right]$ satisfying $\operatorname{deg}\left(Q_{i} \cdot R_{i k}\right)=\operatorname{deg}\left(\gamma_{j k} \cdot g_{j k}\right)=N-d \cdot\|I\|$. Let $\widehat{R}_{i k}$, and $\widehat{\gamma}_{j k}$ be some special representatives of $R_{i k}$, and $\gamma_{j k}$, respectively. Then $\widehat{h}_{k}:=\sum_{i=1}^{n} Q_{i} \cdot \widehat{R}_{i k}+\sum_{j=1}^{m_{k}} g_{j k} \cdot \widehat{\gamma}_{j k}$ is a representative of $h_{k}$. By Lemma 2.3, and since $\mathcal{Q}$ is a $V$ - admissible set, there exists $\alpha \in A$ such that:

i) $\left\{\widehat{h}_{k}(\alpha)\right\}_{k=1}^{K}$ is a basis of $\left(\mathcal{I}(V), Q_{1}, \ldots, Q_{n}\right)_{N-d \cdot\|I\|}(\alpha)$,

ii) all coefficients of $Q_{j}, \hat{R}_{j k}, \widehat{\gamma}_{j k}, g_{j k}$ are well defined at $\alpha$, and

iii) homogeneous polynomials $Q_{0}(\alpha), \ldots, Q_{n}(\alpha) \in k\left[x_{0}, \ldots, x_{M}\right]$ have no common zeros point in $V(\bar{k})$.

On the other hand, it is clear that $\hat{h}_{k}(\alpha) \in\left(\mathcal{I}(V), Q_{1}(\alpha), \ldots, Q_{n}(\alpha)\right)$, for all $k=1, \ldots, K$. Hence, by (2.11), and by i), we have

$$
\left(\mathcal{I}(V), Q_{1}(\alpha), \ldots, Q_{n}(\alpha)\right)_{N-d \cdot\|I\|}=\left(\mathcal{I}(V), Q_{1}, \ldots, Q_{n}\right)_{N-d \cdot\|I\|}(\alpha) .
$$

Then, we have

$$
\begin{aligned}
\operatorname{dim}_{\mathcal{R}_{A, \mathcal{Q}}}\left(\mathcal{I}(V), Q_{1}, \ldots, Q_{n}\right)_{N-d \cdot\|I\|} & =\operatorname{dim}_{k}\left(\mathcal{I}(V), Q_{1}, \ldots, Q_{n}\right)_{N-d \cdot\|I\|}(\alpha) \\
& =\operatorname{dim}_{k}\left(\mathcal{I}(V), Q_{1}(\alpha), \ldots, Q_{n}(\alpha)\right)_{N-d \cdot\|I\|} .
\end{aligned}
$$

This implies that

$$
\begin{aligned}
\operatorname{dim} \frac{\mathcal{R}_{A, \mathcal{Q}}\left[x_{0}, \ldots, x_{M}\right]_{N-d\|I\|}}{\left(\mathcal{I}(V), Q_{1}, \ldots, Q_{n}\right)_{N-d \cdot\|I\|}} & =\operatorname{dim} \frac{k\left[x_{0}, \ldots, x_{M}\right]_{N-d\|I\|}}{\left(\mathcal{I}(V), Q_{1}(\alpha), \ldots, Q_{n}(\alpha)\right)_{N}} \\
& =\operatorname{dim} \frac{\bar{k}\left[x_{0}, \ldots, x_{M}\right]_{N-d\|I\|}}{\left(\mathcal{I}(V(\bar{k})), Q_{1}(\alpha), \ldots, Q_{n}(\alpha)\right)_{N-d \cdot\|I\|}} .
\end{aligned}
$$


On the other hand, by the Hilbert-Serre Theorem ([8], Theorem 7.5), there exist positive integers $n_{1}, c$ such that

$$
\operatorname{dim} \frac{\bar{k}\left[x_{0}, \ldots, x_{M}\right]_{N-d\|I\|}}{\left(\mathcal{I}(V(\bar{k})), Q_{1}(\alpha), \ldots, Q_{n}(\alpha)\right)_{N-d \cdot\|I\|}}=c
$$

for all $I \in \mathbb{N}_{0}^{n}$ and $N \in \mathbb{N}_{0}$ satisfying $N-d\|I\| \geq n_{1}$.

Combining with (2.12), we have

$$
\operatorname{dim} \frac{\mathcal{R}_{A, \mathcal{Q}}\left[x_{0}, \ldots, x_{M}\right]_{N-d\|I\|}}{\left(\mathcal{I}(V), Q_{1}, \ldots, Q_{n}\right)_{N-d \cdot\|I\|}}=c,
$$

for all $I \in \mathbb{N}_{0}^{n}$ and $N \in \mathbb{N}_{0}$ satisfying $N-d\|I\| \geq n_{1}$.

Let $h^{I}$ and $h$ be the Hilbert functions of $\frac{\mathcal{R}_{A, \mathcal{Q}}\left[x_{0}, \ldots, x_{M}\right]}{\mathcal{L}^{I}}$ and $\frac{\mathcal{R}_{A, \mathcal{Q}}\left[x_{0}, \ldots, x_{M}\right]}{\left.\mathcal{I}(V), Q_{1}, \ldots, Q_{n}\right)}$, respectively. Since $\left(\mathcal{I}(V), Q_{1}, \ldots, Q_{n}\right) \subset \mathcal{L}^{I}$, we have $h^{I} \leqslant h$. On the other hand, by Matsumura [11, Theorem 14, $h^{I}(k)$ is a polynomial in $k$ for all $k>>0$ and by (2.12), we have $h(k)=c$ for all $k \geq n_{1}$. Hence, there are constants $m^{I}, n_{2}$ such that $h^{I}(k)=m^{I}$ for all $k \geq n_{2}$ and then $m_{N}^{I}=$ $h^{I}(N-d\|I\|)=m^{I}$ for all $N \in \mathbb{N}_{0}$ satisfying $N-d\|I\| \geq n_{2}$. By Lemma 2.6, we may choose $n_{2}$ common for all $I$. Taking $n_{0}:=\max \left\{n_{1}, n_{2}\right\}$, we get Lemma 2.8, i) and ii).

We have $m_{N}^{I}=h^{I}(N-d\|I\|) \leqslant h(N-d\|I\|) \leqslant \max \{c, h(k): k=$ $\left.0, \ldots, n_{0}\right\}$. Hence, taking $c^{\prime}:=\max \left\{c, h(k): k=0, \ldots, n_{0}\right\}$, we get Lemma 2.8 , iii).

Set

$$
m:=\min _{I \in \mathbb{N}_{0}^{n}} m^{I}
$$

We fix $I_{0}=\left(i_{01}, \ldots, i_{0 n}\right) \in \mathbb{N}_{0}^{n}$, and $N_{0} \in \mathbb{N}_{0}$ such that $N_{0}-d\left\|I_{0}\right\| \geq n_{0}$ and $m_{N_{0}}^{I_{0}}=m$.

For each positive integer $N$, divisible by $d$, denote by $\tau_{N}^{0}$ the set of all $I=\left(i_{1}, \ldots, i_{n}\right) \in \tau_{N}$ such that $N-d\|I\| \geq n_{0}$ and $i_{k} \geq \max \left\{i_{01}, \ldots, i_{0 n}\right\}$, for all $k \in\{1, \ldots, n\}$. 
We have

$$
\begin{gathered}
\# \tau_{N}=\left(\begin{array}{c}
\frac{N}{d}+n \\
n
\end{array}\right)=\frac{1}{d^{n}} \cdot \frac{N^{n}}{n !}+O\left(N^{n-1}\right), \\
\#\left\{I \in \tau_{N}: N-d\|I\| \leqslant n_{0}\right\}=O\left(N^{n-1}\right), \\
\#\left\{I=\left(i_{1}, \ldots, i_{n}\right) \in \tau_{N}: i_{k}<\max _{1 \leqslant \ell \leqslant n} i_{0 \ell}, \text { for some } k\right\}=O\left(N^{n-1}\right), \text { and so } \\
\# \tau_{N}^{0}=\frac{1}{d^{n}} \cdot \frac{N^{n}}{n !}+O\left(N^{n-1}\right) .
\end{gathered}
$$

Similarly to 3], Lemma 2.9, we have:

Lemma 2.9. $m_{N}^{I}=\operatorname{deg} V \cdot d^{n}$ for all $N>>0$, divisible by $d$, and $I \in \tau_{N}^{0}$.

Proof. For any $\gamma \in \mathcal{L}_{N_{0}}^{I^{0}}$, we have

$$
T:=Q_{1}^{i_{01}} \cdots Q_{n}^{i_{0 n}} \gamma-\sum_{E=\left(e_{1}, \ldots, e_{n}\right)>I_{0}} Q_{1}^{e_{1}} \cdots Q_{n}^{e_{n}} \gamma_{E} \in \mathcal{I}_{A, \mathcal{Q}}(V)_{N_{0}},
$$

for some $\gamma_{E} \in \mathcal{R}_{A, \mathcal{Q}}\left[x_{0}, \ldots, x_{M}\right]_{N-d\|E\|}$.

Then, for any $I=\left(i_{1}, \ldots, i_{n}\right) \in \tau_{N}^{0}$, we have

$$
\begin{aligned}
Q_{1}^{i_{1}} \cdots Q_{n}^{i_{n}} \gamma & -\sum_{E=\left(e_{1}, \ldots, e_{n}\right)>I_{0}} Q_{1}^{e_{1}+i_{1}-i_{01}} \cdots Q_{n}^{e_{n}+i_{n}-i_{0 n}} \gamma_{E} \\
& =Q_{1}^{i_{1}-i_{01}} \cdots Q_{n}^{i_{n}-i_{0 n}} \cdot T \in \mathcal{I}_{A, \mathcal{Q}}(V)_{N_{0}} .
\end{aligned}
$$

On the other hand since $I \in \tau_{N}^{0}$ and $E>I_{0}$, we have $\left(e_{1}+i_{1}-i_{01}, \ldots, e_{n}+\right.$ $\left.i_{n}-i_{0 n}\right)>I$.

Hence, by (2.15) we have

$$
\gamma \in \mathcal{L}_{N_{0}+d\|I\|-d\left\|I_{0}\right\|}^{I}
$$

This implies that

$$
\mathcal{L}_{N_{0}}^{I_{0}} \subset \mathcal{L}_{N_{0}+d\|I\|-d\left\|I_{0}\right\|}^{I} \cdot
$$

Then

$$
\begin{aligned}
m=m_{N_{0}}^{I_{0}} & =\operatorname{dim}_{\mathcal{R}_{A, \mathcal{Q}}} \frac{\mathcal{R}_{A, \mathcal{Q}}\left[x_{0}, \ldots, x_{M}\right]_{N_{0}-d\left\|I_{0}\right\|}}{\mathcal{L}_{N_{0}}^{I_{0}}} \\
& \geq \operatorname{dim}_{\mathcal{R}_{A, \mathcal{Q}}} \frac{\mathcal{R}_{A, \mathcal{Q}}\left[x_{0}, \ldots, x_{M}\right]_{N_{0}-d\left\|I_{0}\right\|}}{\mathcal{L}_{N_{0}+d\|I\|-d\left\|I_{0}\right\|}^{I}} \\
& =m_{N_{0}+d\|I\|-d\left\|I_{0}\right\|}^{I} \cdot
\end{aligned}
$$


On the other hand since $\left(N_{0}+d\|I\|-d\left\|I_{0}\right\|\right)-d\|I\|=N_{0}-d\left\|I_{0}\right\| \geq n_{0}$, and $N-\|I\| \geq n_{0}$ (note that $I \in \tau_{N}^{0}$ ), by Lemma 2.8, we have

$$
m^{I}=m_{N_{0}+d\|I\|-d\left\|I_{0}\right\|}^{I}=m_{N}^{I} .
$$

Hence, by (2.16), $m \geq m^{I}=m_{N}^{I}$. Then, by the minimum property of $m$, we get that

$$
m_{N}^{I}=m \text { for all } I \in \tau_{N}^{0}
$$

We now prove that:

$$
\operatorname{dim}_{\mathcal{R}_{A, \mathcal{Q}}} \mathcal{I}_{A, \mathcal{Q}}(V)_{N}=\operatorname{dim}_{k} \mathcal{I}(V)_{N}
$$

Indeed, let $\left\{P_{1}, \ldots, P_{s}\right\}$ be a basis of the $k$ vector space $\mathcal{I}(V)_{N}$. It is clear that $\mathcal{I}_{\mathcal{R}_{A, \mathcal{Q}}}(V)_{N}$ is a vector space over $\mathcal{R}_{A, \mathcal{Q}}$ generated by $\mathcal{I}(V)_{N}$, therefore $\left\{P_{1}, \ldots, P_{s}\right\}$ is also a generating system of $\mathcal{I}_{A, \mathcal{Q}}(V)_{N}$. Then, for (2.18), it suffices to prove that if $t_{1}, \ldots, t_{s} \in \mathcal{R}_{A, \mathcal{Q}}$ satisfy

$$
t_{1} \cdot P_{1}+\cdots+t_{s} \cdot P_{s} \equiv 0
$$

then $t_{1}=\cdots=t_{s} \equiv 0$. We rewrite (2.19) in the following form

$$
C \cdot\left(\begin{array}{c}
t_{1} \\
\cdot \\
\cdot \\
\cdot \\
t_{s}
\end{array}\right)=\left(\begin{array}{c}
0 \\
\cdot \\
\cdot \\
\cdot \\
0
\end{array}\right),
$$

where $C \in \operatorname{Mat}\left(\left(\begin{array}{c}M+N \\ N\end{array}\right) \times s, \mathcal{R}_{A, \mathcal{Q}}\right)$.

If the above system of linear equations has non-trivial solutions, then $\operatorname{rank}_{\mathcal{R}_{A, \mathcal{Q}}} C<$ $s$. Then $\operatorname{rank}_{k} C(z)<s$ for all, but finitely many $z \in A$. Taking $a \in A$ such that $\operatorname{rank}_{k} C(a)<s$. Then the following system of linear equations

$$
C(a) \cdot\left(\begin{array}{c}
t_{1} \\
\cdot \\
\cdot \\
\cdot \\
t_{s}
\end{array}\right)=\left(\begin{array}{c}
0 \\
\cdot \\
\cdot \\
\cdot \\
0
\end{array}\right)
$$


has some non-trivial solution $\left(t_{1}, \ldots, t_{s}\right)=\left(\alpha_{1}, \ldots, \alpha_{s}\right) \in k^{s} \backslash\{0\}$. Then $\alpha_{1} \cdot P_{1}+\cdots+\alpha_{s} \cdot P_{s} \equiv 0$, this is a contradiction. Hence, we get (2.18) .

By Lemma 2.7 and (2.18), we have

$$
\begin{aligned}
\sum_{I \in \tau_{N}} m_{N}^{I}=\operatorname{dim}_{\mathcal{R}_{A, \mathcal{Q}}} \frac{\mathcal{R}_{A, \mathcal{Q}}\left[x_{0}, \ldots, x_{M}\right]_{N}}{\mathcal{I}_{A, \mathcal{Q}}(V)_{N}} & =\operatorname{dim}_{k} \frac{k\left[x_{0}, \ldots, x_{M}\right]_{N}}{\mathcal{I}(V)_{N}} \\
& =\operatorname{dim}_{\bar{k}} \frac{\bar{k}\left[x_{0}, \ldots, x_{M}\right]_{N}}{\mathcal{I}(V(\bar{k}))_{N}} \\
& =\operatorname{deg} V \cdot \frac{N^{n}}{n !}+O\left(N^{n-1}\right)
\end{aligned}
$$

for all $N$ large enough.

Combining with (2.17), we have

$$
m \cdot \# \tau_{N}^{0}+\sum_{I \in \tau_{N} \backslash \tau_{N}^{0}} m_{N}^{I}=\operatorname{deg} V \cdot \frac{N^{n}}{n !}+O\left(N^{n-1}\right) .
$$

On the other hand by Lemma 2.8, $m_{N}^{I} \leqslant c^{\prime}$, for all $I \in \tau_{N} \backslash \tau_{N}^{0}$. Hence, by (2.14), we have

$$
m=\operatorname{deg} V \cdot d^{n}
$$

Combining with (2.17), we have

$$
m_{N}^{I}=\operatorname{deg} V \cdot d^{n}
$$

for all $I \in \tau_{N}^{0}$.

Similarly to [3], Lemma 2.10, we have

Lemma 2.10. For each $s \in\{1, \ldots, n\}$, and for $N>>0$, divisible by $d$, we have:

$$
\sum_{I=\left(i_{1}, \ldots, i_{n}\right) \in \tau_{N}} m_{N}^{I} \cdot i_{s} \geq \frac{\operatorname{deg} V}{d \cdot(n+1) !} N^{n+1}-O\left(N^{n}\right)
$$

Proof. Firstly, we note that if $I=\left(i_{1}, \ldots, i_{n}\right) \in \tau_{N}^{0}$, then all symmetry $I^{\prime}=$ $\left(i_{\sigma(1)}, \ldots, i_{\sigma(n)}\right)$ of $I$ also belongs to $\tau_{N}^{0}$. On the other hand, by Lemma 2.9, 
we have $m_{N}^{I}=\operatorname{deg} V \cdot d^{n}$, for all $I \in \tau_{N}^{0}$. Therefore, by (2.14) we have

$$
\begin{aligned}
\sum_{I=\left(i_{1}, \ldots, i_{n}\right) \in \tau_{N}^{0}} & m_{N}^{I} \cdot i_{1}=\cdots=\sum_{I=\left(i_{1}, \ldots, i_{n}\right) \in \tau_{N}^{0}} m_{N}^{I} \cdot i_{n} \\
& =\operatorname{deg} V \cdot d^{n} \cdot \sum_{I \in \tau_{N}^{0}} \frac{\|I\|}{n} \\
& =\operatorname{deg} V \cdot d^{n} \cdot\left(\sum_{I \in \tau_{N}} \frac{\|I\|}{n}-\sum_{I \in \tau_{N} \backslash \tau_{N}^{0}} \frac{\|I\|}{n}\right) \\
& \geq \operatorname{deg} V \cdot d^{n}\left(\sum_{k=0}^{\frac{N}{d}} \frac{k}{n} \cdot\left(\begin{array}{c}
k+n-1 \\
n-1
\end{array}\right)-\left(\# \tau_{N}-\# \tau_{N}^{0}\right) \cdot \frac{N}{n d}\right) \\
& =\operatorname{deg} V \cdot d^{n}\left(\begin{array}{c}
\left.\sum_{k=0}^{\frac{N}{d}} \frac{k}{n} \cdot\left(\begin{array}{c}
k+n-1 \\
n-1
\end{array}\right)-O\left(N^{n-1}\right) \cdot \frac{N}{n d}\right) \\
\end{array}\right. \\
& =\operatorname{deg} V \cdot d^{n} \sum_{k=1}^{\frac{N}{d}}\left(\begin{array}{c}
k+n-1 \\
n
\end{array}\right)-O\left(N^{n}\right) \\
& \geq \frac{\operatorname{deg} V \cdot d^{n}\left(\begin{array}{c}
\frac{N}{d}+n \\
n+1
\end{array}\right)-O\left(N^{n}\right)}{d \cdot(n+1) !} N^{n+1}-O\left(N^{n}\right) .
\end{aligned}
$$

Hence, for each $i \in\{1, \ldots, n\}$

$$
\begin{aligned}
\sum_{I=\left(i_{1}, \ldots, i_{n}\right) \in \tau_{N}} m_{N}^{I} \cdot i_{s} & \geq \sum_{I=\left(i_{1}, \ldots, i_{n}\right) \in \tau_{N}^{0}} m_{N}^{I} \cdot i_{s} \\
& \geq \frac{\operatorname{deg} V}{d \cdot(n+1) !} N^{n+1}-O\left(N^{n}\right) .
\end{aligned}
$$

We recall that by (2.20), for $N>>0$, we have

$$
\operatorname{dim}_{\mathcal{R}_{A, \mathcal{Q}}} \frac{\mathcal{R}_{A, \mathcal{Q}}\left[x_{0}, \ldots, x_{M}\right]_{N}}{\mathcal{I}_{A, \mathcal{Q}}(V)_{N}}=H_{V}(N)=\operatorname{deg} V \cdot \frac{N^{n}}{n !}+O\left(N^{n-1}\right) .
$$

Therefore, from Lemmas 2.7, 2.10 we get immediately the following result. 
Lemma 2.11. For all $N>>0$ divisible $d$, there are homogeneous polynomials $\phi_{1}, \ldots, \phi_{H_{V}(N)}$ in $\mathcal{R}_{A, \mathcal{Q}}\left[x_{0}, \ldots, x_{M}\right]_{N}$ such that they form a basis of the $\mathcal{R}_{A, \mathcal{Q}}-$ vector space $\frac{\mathcal{R}_{A, \mathcal{Q}}\left[x_{0}, \ldots, x_{M}\right]_{N}}{\mathcal{I}_{A, \mathcal{Q}}(V)_{N}}$, and

$$
\prod_{j=1}^{H_{V}(N)} \phi_{j}-\left(Q_{1} \cdots Q_{n}\right)^{\frac{\operatorname{deg} V \cdot N^{n+1}}{d \cdot(n+1) !}-u(N)} \cdot P \in \mathcal{I}_{A, \mathcal{Q}}(V)_{N}
$$

where $u(N)$ is a function satisfying $u(N) \leqslant O\left(N^{n}\right), P \in \mathcal{R}_{A, \mathcal{Q}}\left[x_{0}, \ldots, x_{M}\right]$ is a homogeneous polynomials of degree

$$
N \cdot H_{V}(N)-\frac{n \cdot \operatorname{deg} V \cdot N^{n+1}}{(n+1) !}+u(N)=\frac{\operatorname{deg} V \cdot N^{n+1}}{(n+1) !}+O\left(N^{n}\right) .
$$

\section{Proof of our main theorem}

Proof. By Lemma 2.1 in [2], there exists an infinite index subset $A \subset \Lambda$ which is coherent with respect to $\mathcal{Q}$. By Remark 1.5, we may assume that the polynomials $Q_{j}$ 's have the same degree $d \geq 1$ and their coefficients belong to the field $\mathcal{R}_{A, \mathcal{Q}}$. By the fact that for any infinite subset $B$ of $A$, then $B$ is also coherent with respect to $\mathcal{Q}$ and $\mathcal{R}_{B, \mathcal{Q}} \subset \mathcal{R}_{A, \mathcal{Q}}$, in our proof, we may freely pass to infinite subsets. For simplicity, we still denote these infinite subsets by $A$.

From the assumption, for each $a \in \mathcal{R}_{A, \mathcal{Q}}$, and $v \in M(k)$, we have, for all $\alpha \in A$,

$$
\log \|a(\alpha)\|_{v} \leq \sum_{v \in M(k)} \log ^{+}\|a(\alpha)\|_{v}=h(a(\alpha)) \leq o(h(x(\alpha)))
$$

For each $v \in S$, and $\alpha \in A$, there exist a subset $J(v, \alpha)=\left\{j_{1}(v, \alpha), \ldots, j_{n}(v, \alpha)\right\} \subset$ $\{1, \ldots, q\}$ such that

$$
\begin{aligned}
0<\left\|Q_{j_{1}(v, \alpha)}(\alpha)(x(\alpha))\right\|_{v} \leq \ldots & \leq\left\|Q_{j_{n}(v, \alpha)}(\alpha)(x(\alpha))\right\|_{v} \\
& \leq \min _{j \notin\left\{j_{1}(v, \alpha), \ldots, j_{n}(v, \alpha)\right\}}\left\|Q_{j}(\alpha)(x(\alpha))\right\|_{v} .
\end{aligned}
$$


By Lemma 2.1, we have

$$
\begin{aligned}
\log \prod_{j=1}^{q}\left\|Q_{j}(\alpha)(x(\alpha))\right\|_{v} & =\log \prod_{\beta_{j} \notin J(v, \alpha)}\left\|Q_{\beta_{j}}(\alpha)(x(\alpha))\right\|_{v}+\log \prod_{i=1}^{n}\left\|Q_{j_{i}(v, \alpha)}(\alpha)(x(\alpha))\right\|_{v} \\
& \geq(q-n) d \log \|x(\alpha)\|_{v}-\log \tilde{h}_{v}(x(\alpha)) \\
& +\log \prod_{i=1}^{n}\left\|Q_{j_{i}(v, \alpha)}(\alpha)(x(\alpha))\right\|_{v}
\end{aligned}
$$

where $\tilde{h}_{v}=\prod\left(1+h_{\mu}\right), h_{\mu}$ runs over all the choices of $l_{2, v}$, thus $\tilde{h}_{v} \in \mathcal{C}_{x}$.

By Lemma 2.11, there exist homogeneous polynomials $\phi_{1}^{J(v, \alpha)}, \ldots, \phi_{H_{V}(N)}^{J(v, \alpha)}$ (depend on $J(v, \alpha))$ in $\mathcal{R}_{A, \mathcal{Q}}\left[x_{0}, \ldots, x_{M}\right]_{N}$ and there are functions $u(N), v(N)$ (com-

mon for all $J(v, \alpha))$ such that $\left\{\phi_{i}^{J(v, \alpha)}\right\}$ a basic of $\mathcal{R}_{A, \mathcal{Q}}-$ vector space $\frac{\mathcal{R}_{A, \mathcal{Q}}\left[x_{0}, \ldots, x_{M}\right]_{N}}{\mathcal{I}_{A, \mathcal{Q}}(V)_{N}}$ and

$$
\prod_{\ell=1}^{H_{V}(N)} \phi_{\ell}^{J(v, \alpha)}-\left(Q_{j_{1}(v, \alpha)} \ldots Q_{j_{n}(v, \alpha)}\right)^{\frac{\operatorname{deg} V \cdot N^{n+1}}{d(n+1) !}-u(N)} P_{J(v, \alpha)} \in \mathcal{I}_{A, \mathcal{Q}}(V)_{N},
$$

where $P_{J(v, \alpha)} \in \mathcal{R}_{A, \mathcal{Q}}\left[x_{0}, \ldots, x_{M}\right]$ is a homogeneous polynomials of degree $\frac{\operatorname{deg} V \cdot N^{n+1}}{(n+1) !}+v(N)$.

Thus, for all $x(\alpha) \in V(k)$, we have

$$
\prod_{\ell=1}^{H_{V}(N)} \phi_{\ell}^{J(v, \alpha)}(\alpha)(x(\alpha))=\left(\prod_{i=1}^{n} Q_{j_{i}(v, \alpha)}(\alpha)(x(\alpha))\right)^{\frac{\operatorname{deg} V \cdot N^{n+1}}{d(n+1) !}-u(N)} P_{J(v, \alpha)}(\alpha)(x(\alpha)) .
$$

On the other hand, it is easy to that there exist $h_{J(v, \alpha)} \in \mathcal{C}_{x}$ such that

$$
\begin{aligned}
\left\|P_{J(v, \alpha)}(\alpha)(x(\alpha))\right\|_{v} & \leqslant\|(x(\alpha))\|_{v}^{\operatorname{deg} P_{J(v, \alpha)}} h_{J(v, \alpha)}(\alpha) \\
& =\|(x(\alpha))\|_{v}^{\frac{\operatorname{deg} V \cdot N^{n+1}}{(n+1) !}+v(N)} h_{J(v, \alpha)}(\alpha) .
\end{aligned}
$$

Therefore,

$$
\begin{aligned}
\log \prod_{\ell=1}^{H_{N}(V)}\left\|\phi_{\ell}^{J(v, \alpha)}(\alpha)(x(\alpha))\right\|_{v} & \leqslant\left(\frac{\operatorname{deg} V \cdot N^{n+1}}{d(n+1) !}-u(N)\right) \cdot \log \left\|\prod_{i=1}^{n} Q_{j_{i}(v, \alpha)}(\alpha)(x(\alpha))\right\|_{v} \\
& \left.+\log ^{+} h_{J(v, \alpha)}(\alpha)+\left(\frac{\operatorname{deg} V \cdot N^{n+1}}{(n+1) !}+v(N)\right) \log \| x(\alpha)\right) \|_{v}
\end{aligned}
$$


This implies that there are functions $\omega_{1}(N), \omega_{2}(N) \leqslant O\left(\frac{1}{N}\right)$ such that

$$
\begin{aligned}
\log \left\|\prod_{i=1}^{n} Q_{j_{i}(v, \alpha)}(\alpha)(x(\alpha))\right\|_{v} & \geq\left(\frac{d(n+1) !}{\operatorname{deg} V \cdot N^{n+1}}-\frac{\omega_{1}(N)}{N^{n+1}}\right) \cdot \log \prod_{\ell=1}^{H_{N}(V)}\left\|\phi_{\ell}^{J(v, \alpha)}(\alpha)(x(\alpha))\right\|_{v} \\
& \left.-\log ^{+} \tilde{h}_{J(v, \alpha)}(\alpha)-\left(d+\omega_{2}(N)\right) \log \| x(\alpha)\right) \|_{v},
\end{aligned}
$$

for some $\tilde{h}_{J(v, \alpha)} \in \mathcal{C}_{x}$.

By (3.2) and (3.3), we have

$$
\begin{aligned}
\log \prod_{j=1}^{q}\left\|Q_{j}(\alpha)(x(\alpha))\right\|_{v} & \geq(q-n-1) d \log \|x(\alpha)\|_{v}-\log ^{+} \tilde{h}_{v}(x(\alpha)) \\
& +\left(\frac{d(n+1) !}{\operatorname{deg} V \cdot N^{n+1}}-\frac{\omega_{1}(N)}{N^{n+1}}\right) \cdot \log \prod_{\ell=1}^{H_{N}(V)}\left\|\phi_{\ell}^{J(v, \alpha)}(\alpha)(x(\alpha))\right\|_{v} \\
& \left.-\log ^{+} \tilde{h}_{J(v, \alpha)}(\alpha)-\omega_{2}(N) \log \| x(\alpha)\right) \|_{v} .
\end{aligned}
$$

We fix homogeneous polynomials $\Phi_{1}, \ldots, \Phi_{H_{N}(V)} \in \mathcal{R}_{A,\left\{Q_{j}\right\}_{j=1}^{q}}\left[x_{0}, \ldots, x_{M}\right]_{N}$ such that they form a basic of $\mathcal{R}_{A, \mathcal{Q}}-$ vector space $\frac{\mathcal{R}_{A, \mathcal{Q}}\left[x_{0}, \ldots, x_{M}\right]_{N}}{\mathcal{I}_{A, \mathcal{Q}}(V)_{N}}$. Then, there exist homogeneous linear polynomials

$$
L_{1}^{J(v, \alpha)}, \ldots, L_{H_{N}(V)}^{J(v, \alpha)} \in \mathcal{R}_{A, \mathcal{Q}}\left[y_{1}, \ldots, y_{H_{V}(N)}\right]
$$

such that they are linear independent over $\mathcal{R}_{A,\left\{Q_{j}\right\}_{j=1}^{q}}$ and

$$
\phi_{\ell}^{J(v, \alpha)}-L_{\ell}^{J(v, \alpha)}\left(\Phi_{1}, \ldots, \Phi_{H_{N}(V)}\right) \in \mathcal{I}_{A, \mathcal{Q}}(V)_{N}
$$

for all $\ell=1, \ldots, H_{V}(N)$. It is clear that $h\left(L_{\ell}^{J(v, \alpha)}(\beta)\right)=o(h(x(\beta))), \beta \in A$ and $A$ is coherent with respect to $\left\{L_{\ell}\right\}_{\ell=1}^{H_{N}(V)}$.

We have,

$$
\prod_{\ell=1}^{H_{V}(N)}\left\|\phi_{\ell}^{J(v, \alpha)}(\alpha)(x(\alpha))\right\|_{v}=\prod_{\ell=1}^{H_{N}(V)}\left\|L_{\ell}^{J(v, \alpha)}\left(\Phi_{1}, \ldots, \Phi_{H_{V}(N)}\right)(\alpha)(x(\alpha))\right\|_{v}
$$


We write

$$
L_{\ell}^{J(v, \alpha)}\left(y_{1}, \ldots, y_{H_{V}(N)}\right)=\sum_{s=1}^{H_{V}(N)} g_{\ell r} y_{s}, \quad g_{\ell s} \in \mathcal{R}_{A, \mathcal{Q}} .
$$

Since $L_{1}^{J(v, \alpha)}, \ldots, L_{H_{N}(V)}^{J(v)}$ are linear independent over $\mathcal{R}_{A, \mathcal{Q}}$, we have $\operatorname{det}\left(h_{\ell s}\right) \neq$ $0 \in \mathcal{R}_{A, \mathcal{Q}}$. Thus, due to cohenrent property of $A$, $\operatorname{det}\left(h_{\ell s}\right)(\beta) \neq 0$ for all $\beta \in A$, outside a finite subset of $A$. By passing to an infinite subset if necessary, we may assume that $L_{1}^{J(v, \alpha)}(\beta), \ldots, L_{H_{N}(V)}^{J(v, \alpha)}(\beta)$ are lineraly independent over $k$ for all $\beta \in A$.

Now we consider the sequence of points $F(\alpha)=\left[\Phi_{1}(x(\alpha)), \ldots, \Phi_{H_{N}(V)}(x(\alpha))\right]$ from $A$ to $\mathbb{P}^{H_{V}(N)-1}(k)$ and moving hyperplanes $\mathcal{L}:=\left\{L_{1}^{J(v, \alpha)}, \ldots, L_{H_{N}(V)}^{J(v, \alpha)}\right\}$ in $\mathbb{P}^{H_{V}(N)-1}(k)$, indexed by $A$. We claim that $F$ is linearly nondegenerate with respect to $\mathcal{L}$. Indeed, ortherwise, then there is a linear form $L \in$ $\mathcal{R}_{B, \mathcal{L}}\left[y_{1}, \ldots, y_{H_{N}(V)}\right]$ for some infinite coherent subset $B \subset A$, such that $\left.L(F)\right|_{B} \equiv 0$ in $B$, which contradicts to the assumption that $x$ is algebraically nondegenerate with respect to $\mathcal{Q}$.

By Theorem A, for any $\epsilon>0$, there is an infinite subset of $A$ (common for all $J(v, \alpha)$ ), denoted again by $A$, such that

$$
\sum_{v \in S} \log \prod_{\ell=1}^{H_{V}(N)} \frac{\|F(\alpha)\|_{v}\left\|L_{\ell}^{J(v, \alpha)}(\alpha)\right\|_{v}}{\left\|L_{\ell}^{J(v, \alpha)}(\alpha)(F(\alpha))\right\|_{v}} \leq\left(H_{V}(N)+\varepsilon\right) h(F(\alpha)),
$$

for all $\alpha \in A$.

Combining with (3.4) and (3.5) we have

$$
\begin{aligned}
\sum_{v \in S} \sum_{j=1}^{q} \log \frac{\|x(\alpha)\|_{v}^{d}}{\left\|Q_{j}(\alpha)(x(\alpha))\right\|_{v}} & \left.\leq(n+1) d \sum_{v \in S} \log \|x(\alpha)\|_{v}+\omega_{2}(N) \sum_{v \in S} \log \| x(\alpha)\right) \|_{v} \\
& +\left(\frac{d(n+1) !}{\operatorname{deg} V \cdot N^{n+1}}-\frac{\omega_{1}(N)}{N^{n+1}}\right) \sum_{v \in S} \log \prod_{\ell=1}^{H_{N}(V)} \frac{\|F(\alpha)\|_{v}\left\|L_{\ell}^{J(v, \alpha)}(\alpha)\right\|_{v}}{\left\|L_{\ell}^{J(v, \alpha)}(\alpha)(x(\alpha))\right\|_{v}} \\
& -H_{V}(N)\left(\frac{d(n+1) !}{\operatorname{deg} V \cdot N^{n+1}}-\frac{\omega_{1}(N)}{N^{n+1}}\right) \sum_{v \in S} \log \|F(\alpha)\|_{v} \\
& +o(h(x(\alpha))) .
\end{aligned}
$$

Since the above inequality is independent of the choice of components of 
$x(\alpha)$, we can choose the components of $x(\alpha)$ being $S-$ integers so that

$$
\sum_{v \in S} \log \|x(\alpha)\|_{v}=h(x(\alpha)), \text { and } \sum_{v \in S} \log \|F(\alpha)\|_{v}=h(F(\alpha)) \leq N h(x(\alpha))+o(h(x(\alpha))) .
$$

Combining with (3.7) and (3.6), we obtain

$$
\begin{aligned}
\sum_{v \in S} \sum_{j=1}^{q} \log \frac{\|x(\alpha)\|_{v}^{d}}{\left\|Q_{j}(\alpha)(x(\alpha))\right\|_{v}} & \leq(n+1) d h(x(\alpha))+\omega_{2}(N) h(x(\alpha)) \\
& +\left(\frac{d(n+1) !}{\operatorname{deg} V \cdot N^{n+1}}-\frac{\omega_{1}(N)}{N^{n+1}}\right)\left(H_{V}(N)+\varepsilon\right) h(F(\alpha)) \\
& -H_{V}(N)\left(\frac{d(n+1) !}{\operatorname{deg} V \cdot N^{n+1}}-\frac{\omega_{1}(N)}{N^{n+1}}\right) h(F(\alpha)) \\
& +o(h(x(\alpha)) .
\end{aligned}
$$

Hence, we obtain

$$
\begin{aligned}
\sum_{v \in S} \sum_{j=1}^{q} \log \frac{\|x(\alpha)\|_{v}^{d}\left\|Q_{j}(\alpha)\right\|_{v}}{\left\|Q_{j}(\alpha)(x(\alpha))\right\|_{v}} & \leq(n+1) d h(x(\alpha))+\omega_{2}(N) h(x(\alpha)) \\
& +\epsilon\left(\frac{d(n+1) !}{\operatorname{deg} V \cdot N^{n+1}}-\frac{\omega_{1}(N)}{N^{n+1}}\right) h(F(\alpha))+o(h(x(\alpha))) .
\end{aligned}
$$

Here, we note that $h\left(Q_{j}(\alpha)\right)=o(h(x(\alpha)))$.

Combining with (3.8), by our choice with $\omega_{1}, \omega_{2}$ for $N$ large enough, we get

$$
\sum_{v \in S} \sum_{j=1}^{q} \log \frac{\|x(\alpha)\|_{v}^{d}\left\|Q_{j}(\alpha)\right\|_{v}}{\left\|Q_{j}(\alpha)(x(\alpha))\right\|_{v}} \leq(n+1+\varepsilon) d h(x(\alpha)),
$$

for all $\alpha \in A$. This completes the proof of Theorem 1.4 ,

\section{References}

[1] Z. Chen, M. Ru, and Q. Yan, The degenerated second main theorem and Schmidt's subspace theorem, Science China, 55 (2012), 1367-1380. 
[2] Z. Chen, M. Ru, and Q. Yan, Schmidt's subspace theorem with moving targets, Int. Math. Res. Not., first published online, August 6, 2014.

[3] W. Cherry, G. Dethloff, and T. V. Tan, Holomorphic curves into algebraic varieties intersecting moving hypersurface targets, arXiv:1503.08801v2 [math.CV] .

[4] P. Corvaja and U. Zannier, On a general Thue's equation, Amer. J. Math. 126 (2004), 1033-1055.

[5] G. Dethloff and T. V. Tan, A second main theorem for moving hypersurface targets, Houston J. Math. 37 (2011), 79-111.

[6] J. H. Evertse and R. G. Ferretti, Diophantine inequalities on projective varieties, Internat. Math. Res. Notices, 25 (2002), 1295-1330.

[7] J. H. Evertse and R. G. Ferretti, A generalization of the subspace theorem with polynomials of higher degree, Developments in Mathematics, 16 (2008), 175-198, Springer-Verlag, New York.

[8] R. Hartshorne, Algebraic Geometry, Grad. Texts in Math. vol 52, Springer-Verlag, New York, 1977.

[9] G. Le, Schmidt's subspace theorem with moving hypersurfaces, Int. J. Number Theory, 11 (2015), 139-158.

[10] A. Levin, On a Schmidt subspace theorem for algebraic points, Duke Math. J. 163 (2014), 2841-2885.

[11] H Matsumura, Commutative Algebra, Benjamin/Cummings Publication Company, Massachusetts, 1980.

[12] M. Ru, A defect relation for holomorphic curves intersecting hypersurfaces, Amer. J. Math. 126 (2004), 215-226.

[13] M. Ru, Holomorphic curves into algebraic varieties, Ann. of Math. 169 (2009), 255-267.

[14] M. Ru and P. Vojta Schmidt's subspace theorem with moving targets, Invent. Math. 127 (1997), 51-65. 
[15] P. Vojta, Diophantine approximation and Nevanlinna theory, in : J.L. Colliot-Thélène, P. Swinnerton-Dyer and P. Vojta (eds.), Arithmetic geometry, 111-124. LNM 2009, Springer-Verlag, Berlin, 2010.

[16] O. Zariski, Generalized weight properties of the resultant of $n+1$ polynomials in $n$ indeterminates, Trans. AMS, 41 (1937), 249-265.

Nguyen Thanh Son

Department of Mathematics

Hanoi National University of Education

136-Xuan Thuy street, Cau Giay, Hanoi, Vietnam

e-mail: k16toannguyenthanhson@gmail.com

Tran Van Tan

Department of Mathematics

Hanoi National University of Education

136-Xuan Thuy street, Cau Giay, Hanoi, Vietnam

e-mail: tranvantanhn@yahoo.com

Nguyen Van Thin

Department of Mathematics

Thai Nguyen University of Education

Luong Ngoc Quyen Street, Thai Nguyen city, Vietnam.

e-mail: thinmath@gmail.com 